\title{
Being Mindful of Perfectionism and Performance Among Athletes in a Judged Sport
}

\author{
Erika D. Van Dyke \\ edv0001@mix.wvu.edu
}

Follow this and additional works at: https://researchrepository.wvu.edu/etd

Part of the Other Psychology Commons

\section{Recommended Citation}

Van Dyke, Erika D., "Being Mindful of Perfectionism and Performance Among Athletes in a Judged Sport" (2019). Graduate Theses, Dissertations, and Problem Reports. 7428.

https://researchrepository.wvu.edu/etd/7428

This Dissertation is protected by copyright and/or related rights. It has been brought to you by the The Research Repository @ WVU with permission from the rights-holder(s). You are free to use this Dissertation in any way that is permitted by the copyright and related rights legislation that applies to your use. For other uses you must obtain permission from the rights-holder(s) directly, unless additional rights are indicated by a Creative Commons license in the record and/ or on the work itself. This Dissertation has been accepted for inclusion in WVU Graduate Theses, Dissertations, and Problem Reports collection by an authorized administrator of The Research Repository @ WVU.

For more information, please contact researchrepository@mail.wvu.edu. 
Being Mindful of Perfectionism and Performance Among Athletes in a Judged Sport

Erika D. Van Dyke

\author{
Dissertation submitted \\ to the College of Physical Activity and Sport Sciences \\ at West Virginia University \\ in partial fulfillment of the requirements for the degree of \\ Doctor of Philosophy in \\ Sport, Exercise, and Performance Psychology
}

Sam J. Zizzi, Ed.D., Chair

Scott Barnicle, Ph.D.

Edward F. Etzel, Ed.D.

Aaron Metzger, Ph.D.

Department of Sport Sciences

Morgantown, West Virginia

2019

Keywords: mindfulness, perfectionism, performance, personality, elite athletes, sport psychology

Copyright 2019 Erika D. Van Dyke 


\author{
ABSTRACT \\ Being Mindful of Perfectionism and Performance Among Athletes in a Judged Sport \\ Erika D. Van Dyke
}

Literature pertaining to mindfulness and perfectionism in sport has expanded greatly in recent years. However, little research has integrated mindfulness and perfectionism, particularly within sports where athletes are judged on performance to a standard of perfection. The current study had two primary aims: (1) to explore profiles of mindfulness and perfectionism among intercollegiate gymnasts through a person-centered approach, and (2) to analyze differences in objective performance measures across the resulting profiles. The analytic sample consisted of 244 NCAA gymnasts representing NCAA Division I, II, and III institutions. Gymnasts completed self-report measures of mindfulness and perfectionism. Competitive performance records (i.e., national qualifying scores) were then gathered for participating gymnasts. Cluster analyses revealed a three-cluster solution: a moderate mindfulness, high perfectionism profile; a low mindfulness, low/moderate perfectionism profile; and a high mindfulness, very low perfectionism profile. Although competitive performance differences were not observed among the three profiles, exploratory post hoc pairwise comparisons indicated potential performance differences on vault and bars. Interestingly, gymnasts in different profiles performed more favorably on each event. Small to moderate effect size estimates provide some evidence that perfectionism may be adaptive to gymnastics performance. Elite level gymnasts were represented across three distinct profiles, suggesting that more than one profile of characteristics may be adaptive for reaching high levels of performance. Further, the sport context might be considered when interpreting the practical significance of the findings. The results can be used to help coaches, researchers, and practitioners better understand how mindfulness and perfectionism are expressed among athletes in a judged sport, and how these tendencies may be impactful in different ways. Future research exploring determinants of performance and mental health concurrently could provide further understanding of whether the characteristics that facilitate performance are congruent with those that facilitate wellbeing. 


\section{Acknowledgements}

Many people have crossed the path of my life during this project, and I will be forever grateful for the indelible footprints they have left there. Some of those people have simply left deeper imprints than others. These words acknowledge my gratitude for your presence on my journey.

I would like to share an immense amount of appreciation for my advisor, Dr. Sam Zizzi. Thank you for extending me an invitation to join this esteemed doctoral program. Your supportive guidance and mentorship throughout this climb up research mountain has been invaluable, and has allowed me to grow as a scholar and more importantly as a person. Thank you for always inviting my curiosity, for your patience with my "thorough" process, for your white board check boxes of accountability, and for your gentle nudges to step back and get some perspective every so often. You have a remarkable way of seeing each of your students for who they are as learners. Thank you for seeing me. You have helped me to embrace the value in letting go, in imperfection, and in embracing my sunshine. Thank you for walking every step of the path along with me. The view from the top of this climb is spectacular.

I would also like to thank Dr. Aaron Metzger for his incredible knowledge of the world of multivariate statistics. You provided the foundational learning that inspired many of the statistical directions taken throughout the course of this research project. Thank you for challenging me to explore interesting and complex questions, for making statistics accessible, and for giving me the skills and confidence necessary to carry out the analyses contained within these pages. I so appreciate you joining me for this glimpse into the experiences of athletes.

My dissertation committee members, Dr. Etzel and Dr. Barnicle, have been such wonderful sources of support throughout this project and more broadly throughout my time here in the halls of CPASS. Thank you, Dr. Etzel, for greeting me each day with, "Ah little miss sunshine." Your endearing reminders to "be well, and do good work," to "walk between the raindrops," and to know that "we'll keep the light on for you" always made me smile and feel a valued member of our learning community. Your lovely words of wisdom and encouragement have and continue to mean so much to me. I am grateful to have been among the many students whose lives you have touched. Thank you, Dr. Barnicle, for always keeping your door open to my many many questions - often multiple in a given day. You have been such a supportive mentor, both in this project and in my growth as a teacher and consultant. I appreciate you being there for me throughout this learning journey.

I am forever grateful that Dr. Zizzi crossed our paths, Candice (Clay) Brown. Thank you so incredibly much for the generous time and energy that you devoted to helping with my research project. From thoughtfully entering and checking data, to playing with statistical analyses, to reading drafts of the document, to presenting a poster together during CPASS research day. Your enthusiasm, dedication, and desire to learn and grow is inspiring, and I appreciate you lending some of your sparkle to our collaboration. Your presence throughout this project has been a blessing, and has brought me the joy of beginning my journey as a mentor. Thank you for your support. I am so excited to see what your very bright future will hold. 
Thank you to all of the faculty in our Sport, Exercise, and Performance Psychology program for your belief in me, and for giving me the opportunity to pursue this path. One of the things that drew me here to WVU was the learning community cultivated by faculty and embraced by students in our program. I appreciate the legacy that you have built, and am so glad to have had the opportunity to be a part of this place.

To my SEPP family. Thank you for your endless support, encouragement, smiles, and hugs throughout this journey, especially on days when the process felt daunting. A special note of appreciation for my cohort members, Adam, Carra, and Seth. What an amazing group of people with whom to share this experience. I am so grateful to have created so many memories with each of you - thank you for touching my life. I will continue to do my best to supply the sunshine and rainbows.

A very special thank you must be extended to the roommates who have helped make this place feel like home during these past four years. Tammy, you led the way and I can only hope to follow in your footsteps to become a real professor someday. Carra, I am beyond grateful to be doing this journey along with you. Thank you for sharing all the laughs, tears, and moments of growth with me. I will forever remember our road trip to New York just after our first semester as doctoral students, and making a pact to do this thing together all the way through. We are so close now \#HancockPact. Matt and Jordan (and Hunter), thank you for welcoming me into your little family these past three years, and for making Morgantown feel a little more like Northern California. I love you all.

Thank you to my partner, Ben, for his love, laughter, and support. What a ride this has been! Whether living together or at a distance, your belief in me has shaped the course of this journey in a beautiful way. I appreciate you for your love of learning, endless curiosity, and for always being there by my side to go chasing waterfalls. Here's to the adventure ahead.

On a final note, to the people who gave me life, who instilled in me a joy of learning, and who have been my foundation through it all - my parents, Ivan and Anne Marie. I appreciate your unconditional love and support of my aspirations more than these simple words can express. Thank you for always believing that I can accomplish anything I set my mind and heart toward. You two are the best cheerleaders and consultants a daughter could ever wish for. I am so lucky you picked me.

"And now that you don't have to be perfect, you can be good." - John Steinbeck 


\section{Table of Contents}

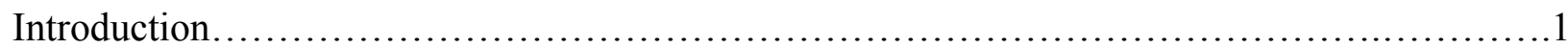

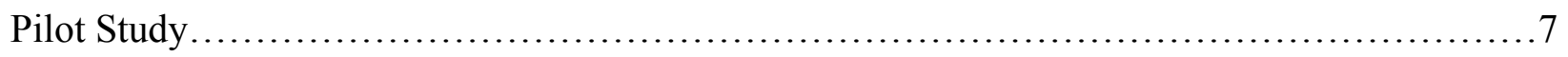

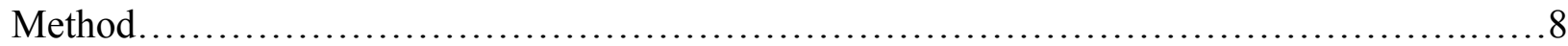

Research Design and Sampling ............................................ 8

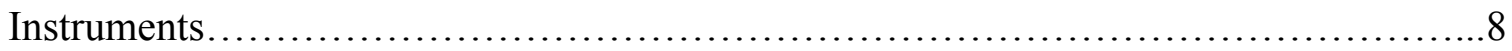

Mindfulness..........................................................

Perfectionism...................................................... 9

Demographic Questionnaire......................................... 10

Competitive Gymnastics Performance................................. 10

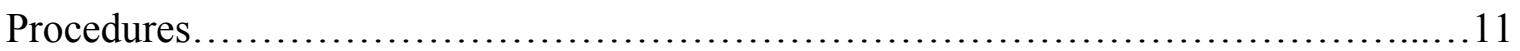

Statistical Analyses......................................................11

Results...........................................................................

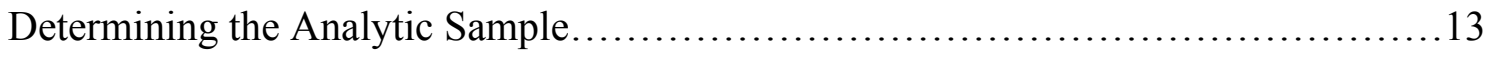

Descriptive Statistics on the Analytic Sample...................................14

Cluster Interpretation................................................. 15

Comparing Performance Across Clusters....................................16

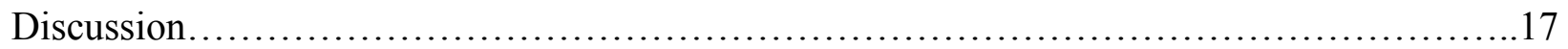

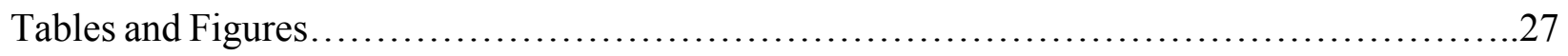

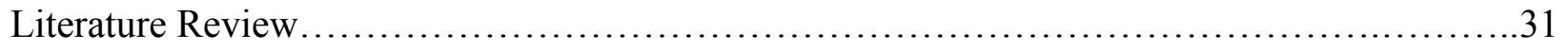

Mindfulness: A Brief Backdrop................................................ 31

Historical Roots.................................................... 32

Mindfulness-Based Approaches........................................33 
Mindfulness in Sport and Performance Psychology ............................... 34

Mindfulness-Based Approaches in Sport......................................35

Linking Mindfulness-Based and Traditional PST Approaches..................37

Mindfulness, Performance, and Psychological Aspects of Sport................39

Perfectionism in Sport and Performance Psychology .............................56

Measurement................................................................66

Mindfulness..........................................................66

Perfectionism...................................................... 70

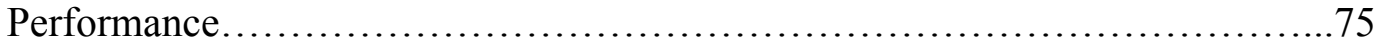

Directions for Future Research................................................. 77

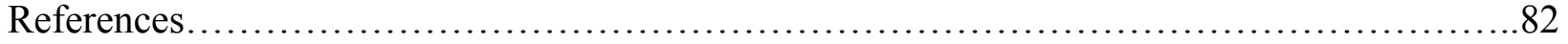

Table 1: Mindfulness Measurements...............................................95

Table 2: Perfectionism Measurements..............................................96

Table 3: Adapted Mindfulness-Flow-Performance Model in Sport...........................97

Appendix A: Athlete Mindfulness Questionnaire $\ldots \ldots \ldots \ldots \ldots \ldots \ldots \ldots \ldots \ldots \ldots \ldots \ldots \ldots \ldots \ldots . . .98$

Appendix B: Sport Multidimensional Perfectionism Scale-2 ........................... 100

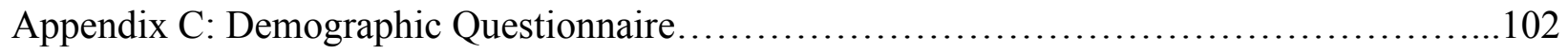

Appendix D: Email to Gymnastics Coaches........................................... 104

Appendix E: Cover Letter and Informed Consent................................... 105

Appendix F: IRB Approval..................................................... 106 


\section{Introduction}

Researchers studying mindfulness and acceptance-based evidence among athletes support a cautious yet optimistic view regarding the efficacy of such approaches in the context of sport (e.g., McAlarnen \& Longshore, 2017; Noetel, Ciarrochi, Van Zanden, \& Lonsdale, 2017; Sappington \& Longshore, 2015). In a recent systematic review, Noetel et al. (2017) analyzed over 60 studies of mindfulness and acceptance-based approaches intended to promote positive sport outcomes, including athletic performance. Despite finding large effect sizes for the performance benefits of such interventions, the findings were deemed low in quality, lacking precision in effect sizes and consistency. Among the individual studies reviewed, researchers found preliminary support for mindfulness and acceptance-based interventions across a variety of sport outcomes (e.g., performance, flow, present-moment awareness, confidence, injury prevention, competitive anxiety, and burnout). Continued research efforts with increased rigor are thus needed to support mindfulness as an approach for enhancing sport performance and related outcomes.

At their core, mindfulness and acceptance-based approaches focus on modifying the relationship one has with internal experiences (e.g., physical sensations, emotions, cognitions), rather than deliberately aiming to change, consciously control, suppress, or reduce internal experiences (e.g., Gardner \& Moore, 2004, 2007; Hayes, Strosahl, \& Wilson, 1999; Kabat-Zinn, 1982). Such mindfulness approaches cultivate present-focused awareness and attention, a nonjudgmental and accepting approach to situations, openness and curiosity toward experience, and compassion for self and others - elements that comprise the flavor of mindfulness (Zizzi, 2017). Recognizing the applicability of mindfulness-based approaches to the context of sport, 
researchers examined the link between mindfulness and objective measures of sport performance using a variety of study designs.

In support of a mindful approach to facilitating sport performance, researchers found that a greater number of athletes who engaged in a mindfulness and acceptance-based program improved their national performance ranking compared to those who took part in a traditional change-based program (Bernier, Thienot, Codron, \& Fournier, 2009). Authors of another study found that elite shooters in a mindfulness meditation group experienced significant increases in shooting performance (i.e., mean performance score increase from 528 to $544, S D=13$ ) and significant decreases in pre-competition anxiety (i.e., mean salivary cortisol level decrease from 1.33 to $0.66, S D=0.07$ ) from pre- to post-test compared to a control group (John, Verma, \& Khanna, 2011). In a non-intervention study, Gooding and Gardner (2009) found that collegiate athletes' levels of mindfulness significantly predicted basketball free throw shooting percentage in games across the competitive season (i.e., one standard deviation increase in mindfulness scores resulted in a $5.75 \%$ increase in free throw shooting percentage). When competitive experience was controlled for, however, mindfulness no longer arose as a significant predictor of competitive performance. Gooding and Gardner noted that competitive experience and mindfulness may predict sport performance through shared variance. Taken together, researchers suggest that mindfulness may influence objectively measured performance in sport among high level athletes. Further research may provide additional clarity regarding the utility of mindfulness for real-world competitive performance.

As mindfulness research continues to evolve, studies that take a more nuanced approach to studying the mindfulness-performance relationship are needed. These kinds of studies can clarify how, when, and by whom mindfulness could be most useful. In a sport like gymnastics 
for instance, in which athletes are judged to a standard of perfection in their skills and technique, perfectionism may be considered contextually relevant to the study of mindfulness-performance relationships. Thus, the study of mindfulness and perfectionism among judged sport athletes may provide more nuanced insights regarding how these constructs are experienced together among individuals, and how those unique experiences may be related to individual differences in sport performance.

Perfectionism has been defined as "a personality disposition characterized by striving for flawlessness and setting exceedingly high standards for performance, accompanied by tendencies for overly critical evaluations" (Stoeber, 2012, p. 294). Although different frameworks of perfectionism have been proposed in the literature (e.g., Frost, Marten, Lahart, \& Rosenblate, 1990; Hewitt \& Flett, 1991), researchers acknowledge that perfectionism is a multidimensional construct. Stoeber and Otto (2006) provided a way to conceptually integrate different proposed frameworks based on two higher-order dimensions of perfectionism - perfectionistic strivings and perfectionistic concerns. Although a point of some debate among perfectionism researchers in recent years (e.g., Flett \& Hewitt, 2005), perfectionistic strivings have often been considered adaptive and facilitative of performance, whereas perfectionistic concerns have often been considered maladaptive and debilitative of performance in sport (e.g., Gotwals, Stoeber, Dunn \& Stoll, 2012; Stoeber, 2012). In a recent meta-analytic review of multidimensional perfectionism in sport, the researchers further highlighted the complexity of these relationships, noting that perfectionistic concerns seem to be clearly maladaptive, while perfectionistic strivings may be adaptive or maladaptive for athletes (Hill, Mallinson-Howard, \& Jowett, 2018). Considering potential contrasts between elements of perfectionism (e.g., self-critical evaluations) and elements of mindfulness (e.g., acceptance and self-compassion), further research integrating the 
two constructs is needed to understand how mindfulness and perfectionism may interact in athletes' experiences.

Many researchers have examined relationships between perfectionism and competitive sport performance among high level athletes (e.g., Gotwals et al., 2012; Hill et al., 2018). Relatively few researchers, however, have explored the intersection of mindfulness and perfectionism in sport. In their follow-up study of the long-term impact of mindfulness-based programming for sport performance, Thompson, Kaufman, De Petrillo, Glass, and Arnkoff (2011) found significant performance improvements in long-distance runners' mile times from pre- and posttest to follow-up. In addition, the authors found negative associations between performance improvements and aspects of perfectionism, including overall trait perfectionism $(r$ $=.74)$, concern over mistakes $(r=.69)$, and doubts about actions $(r=.75)$. It is important to note that negative relationships are reflected in the positive correlations reported because performance improvement is measured through decreased mile time. Although the links between mindfulness, perfectionism, and performance should be interpreted with care due to the correlational nature of the study, Thompson et al. (2011) support the notion that mindfulness may be related to performance benefits, and that certain dimensions of perfectionism may negatively influence athletic performance.

A recent critical evaluation of mindfulness research has raised questions regarding the varied definitions and measurements of mindfulness found in the literature, as well as the proposed, seemingly unquestioned, benefits of mindfulness without regard for potential adverse effects (Van Dam et al., 2018). Rather than leaping with naïve enthusiasm into the application of mindfulness interventions within a new population, researchers might first aim to understand the typical mindfulness experiences of those athletes. Taking a person-centered analytic approach to 
the study of mindfulness may help bring that explicit attention to salient features of mindfulness among athletes in a specific sport setting. Further, new insights gained about the athletes' mindfulness experiences will be highly influenced by the specific core concepts measured in the selected instruments. Considering the work of Van Dam et al. (2018), researchers are encouraged to clearly define the flavor(s) of mindfulness assessed to enhance interpretability of future research findings.

Person-centered approaches to data analysis allow the researcher to better understand unique profiles of the key constructs measured among individuals. Although person-centered approaches have been used in the study of mindfulness (e.g., Kee \& Wang, 2008) and perfectionism (e.g., Gucciardi, Mahoney, Jalleh, Donovan, \& Parkes, 2012), these constructs have yet to be studied concurrently through cluster analytic approaches. In their person-centered approach to the study of mindfulness in sport, Kee and Wang (2008) identified a four-cluster solution based on university athletes' mindfulness scores (Mindfulness/Mindlessness Scale, MMS; Bodner \& Langer, 2001). Individuals in the profile highest in mindfulness showed the most frequent use of psychological skills in sport. Specifically, athletes in the cluster highest in mindfulness had significantly higher goal setting, positive self-talk, and imagery compared to those in clusters lower in mindfulness characteristics. In addition, the researchers found significant differences in flow dispositions across the four mindfulness clusters. Thus, Kee and Wang identified a stable mindfulness cluster solution, and found significant differences among those mindfulness profiles on the outcome variables of psychological skill use and flow dispositions.

Along this line of person-centered research, Gucciardi et al. (2012) explored profiles of perfectionism within a heterogeneous sample of elite athletes through a cluster analytic 
approach. The researchers found three distinct profiles of perfectionism among athletes based on the Sport Multidimensional Perfectionism Scale (Sport-MPS; Dunn, Causgrove Dunn, \& Syrotuik, 2002): (1) adaptive perfectionists - high personal standards, low concern over mistakes, moderate perceived parent/coach pressures, (2) maladaptive perfectionists - high concern over mistakes and perceived parent/coach pressures, moderate/high personal standards, and (3) non-perfectionists - low personal standards and concern over mistakes, moderate perceived parent/coach pressures. Gucciardi et al. further revealed significant differences in motivational orientations among the perfectionism profiles. Specifically, adaptive perfectionists reported significantly lower levels of fear of failure, performance approach and avoidance goals, and mastery avoidance goals, and significantly higher levels of mastery approach goals than did maladaptive perfectionists. Non-perfectionists reported significantly lower levels of the motivational orientations assessed than did maladaptive perfectionists, and lower levels of mastery approach goals, performance approach goals, and intrinsic motivation than did adaptive perfectionists. The researchers thus supported a stable three cluster conceptualization of perfectionism, and found differences in motivational outcomes across those perfectionism profiles. Gucciardi et al. highlighted that among these elite athletes both adaptive and maladaptive perfectionists had high levels of personal standards, and that it was primarily the presence or absence of overly critical self-evaluations that differentiated maladaptive from adaptive perfectionists, respectively.

Many existing studies that have examined mindfulness and links to performance outcomes have done so following programming and interventions (e.g., John et al., 2011; Thompson et al., 2011). Little research has explored athletes' typical tendencies toward mindfulness and perfectionism qualities, and how together these characteristics may relate to 
performance in unique ways. The current study will thus explore mindfulness, perfectionism, and performance among gymnasts - athletes in a judged sport. Aims of the research will be twofold:

(1) to examine whether unique profiles of mindfulness and perfectionism constructs exist among the athlete participants, and (2) to assess whether objective measures of competitive performance differ across the unique mindfulness and perfectionism profiles.

\section{Pilot Study}

The purpose of the pilot study was to assess the psychometric properties of mindfulness and perfectionism measures, and to explore relationships between mindfulness and perfectionism among intercollegiate gymnasts. Participants were female gymnasts $(N=301)$, ranging in age from 18 to 22 years $(M=19.46, S D=1.20)$, who attended NCAA Division I, II, or III colleges and universities in the United States. Gymnasts completed the Athlete Mindfulness Questionnaire (AMQ; Zhang, Chung, \& Si, 2017), and the personal standards and concern over mistakes subscales of the Sport Multidimensional Perfectionism Scale-2 (i.e., Sport-MPS-2; Gotwals \& Dunn, 2009). Results of the correlational analyses supported theoretically expected associations among constructs (e.g., present-moment attention and awareness, $r=.62$; acceptance and concern over mistakes, $r=-.41$ ), and internal reliability coefficients across the mindfulness and perfectionism subscales ranged from .74 to .88. Confirmatory factor analyses supported the original three-factor structure of the AMQ $[R M S E A=.06, S R M R=.05, C F I=$ .92]. The two-factor perfectionism model did not show good fit to the data. Modification indices were reviewed to evaluate potential model fit improvements. When residual error terms of items in the concern over mistakes factor were allowed to covary, the two-factor model fit improved markedly $[\mathrm{RMSEA}=.08, \mathrm{SRMR}=.08, \mathrm{CFI}=.91]$. Through the pilot study, the research team supported the use of the AMQ among intercollegiate level gymnasts, and provided further 
understanding about relationships between mindfulness and perfectionism among athletes in a judged sport context.

\section{Method}

\section{Research Design and Sampling}

The current study built upon the pilot study to explore mindfulness, perfectionism, and performance using a quantitative, descriptive correlational, research design. Female gymnasts 18 years of age or older attending National Collegiate Athletic Association (NCAA) Division I, II, or III colleges and universities in the United States who took part in the pilot study also participated in the present study. Prior to the pilot study, convenience sampling was used to contact all NCAA collegiate women's gymnastics coaches with available contact information to seek permission to collect data with their teams. Gymnasts who previously completed the survey and reported their name were included in the first part of the study exploring mindfulness and perfectionism profiles. Participants who performed on at least one event during the 2019 competition season from January to April, and for whom a National Qualifying Score (NQS) could be calculated, were included in the second part of the study assessing performance differences among the resulting profiles.

\section{Instruments}

Mindfulness. The Athlete Mindfulness Questionnaire (AMQ; Zhang et al., 2017) was used to measure mindfulness in the current study. The AMQ is a 16-item, 3-factor measure of mindfulness for athletes. Mindfulness is assessed based on the subscales present moment attention (e.g., When I find myself distracted, I gently bring my attention back to my training), awareness (e.g., I am aware that my emotions during training and competition can influence my thinking and behavior), and acceptance (e.g., During training and competition, it doesn't matter 
if the situation is good or bad, I can accept myself for who I am). Items are rated on 5-point Likert scales ranging from 1 (never true) to 5 (always true). Confirmatory factor analysis (CFA) revealed satisfactory fit indices for the 16-item, 3 -factor structure of the instrument, $X^{2}(101)=$ 221.28, $p<.001, C F I=0.95, T L I=0.94, W R M R=1.04, R M S E A=0.06$. Internal consistency reliabilities for the three AMQ subscales ranged from 0.64 to 0.76 . In the present study, internal consistency reliabilities for the subscales were slightly higher, ranging from 0.75 to 0.77 . Convergent validity for the AMQ was supported through significant positive associations between the present moment attention, awareness, and acceptance subscales of the AMQ and mindfulness as measured by the Mindful Attention Awareness Scale (MAAS; Brown \& Ryan 2003). Concurrent validity for the three subscales of the AMQ was also supported through significant negative relationships with burnout and experiential avoidance, and significant positive relationships with well-being, positive affect, and dispositional flow.

Perfectionism. The full version of the Sport Multidimensional Perfectionism Scale-2 (Sport-MPS-2; Gotwals \& Dunn, 2009; Gotwals, Dunn, Causgrove Dunn, \& Gamache, 2010) is a 42-item, 6-factor measure assessing the multidimensional nature of perfectionism in sport. The subscale dimensions include personal standards, concern over mistakes, perceived parental pressure, perceived coach pressure, doubts about actions, and organization. Items are rated on 5point Likert scales ranging from 1 (strongly disagree) to 5 (strongly agree). Athletes are asked to report how they "view certain aspects of their competitive experiences in sport." For the purpose of the present study, two subscales, namely personal standards (e.g., It is important to me that I be thoroughly competent in everything I do in my sport) and concern over mistakes (e.g., I should be upset if I make a mistake in competition), were used to measure the two higher-order constructs of perfectionistic strivings and perfectionistic concerns, respectively. The personal 
standards and concern over mistakes subscales were selected because they have been recommended as indicators of perfectionistic strivings and perfectionistic concerns in sport (Stoeber \& Madigan, 2016). The reliability of the Sport-MPS-2 has been demonstrated among athletes, with internal consistencies of .74 and .79 for the personal standards and concern over mistakes subscales, respectively (Gotwals \& Dunn, 2009). In the current study, internal consistency reliabilities for the personal standards and concern over mistakes subscales were .77 and .88 , respectively.

Demographic questionnaire. The demographic questionnaire assessed participants' age, highest level in gymnastics attained before college, race, and ethnicity. Gymnast participants were asked to report their name, and the college or university they attend so the research team could access their publicly available competition scores from an online platform. Experience and satisfaction working with a sport psychology professional, as well as experience with mindfulness, were also assessed. All identifiable information was held confidential, and all data gathered for the study was reported in aggregate to protect the anonymity of participants.

Competitive gymnastics performance. Measurement of competitive gymnastics performance was based on the National Qualifying Score (NQS). The NQS is used in collegiate gymnastics to determine placement in post-season competition, and is calculated based on the following criteria: (1) three highest away scores on a given event, plus (2) next three highest scores on a given event, whether home or away, (3) drop the highest of the six scores, (4) average the remaining five scores. An NQS was calculated for each gymnast who participated in the study for each event on which she competed during the regular meet season to allow for comparison of mindfulness - perfectionism - performance relationships across events (i.e., vault, uneven bars, balance beam, floor exercise). 


\section{Procedures}

After gaining approval from the Institutional Review Board (IRB) to conduct the research methods necessary for both the pilot and current studies, the researcher contacted college gymnastics coaches through email correspondence to request permission to collect data with their athletes. Coaches had the option to receive either paper copies of the counterbalanced questionnaires via mail, or a Qualtrics link to an online version of the questionnaires via email. Coaches then made the surveys available to their athletes to complete on a voluntary basis. All athletes receiving the survey were initially presented with a cover letter description of the study, and were asked to provide their consent to participate. Survey data were collected prior to, or in the beginning of, the competition season for each participant. All data were reported in aggregate, and any identifying information collected in the surveys was used for the sole purpose of accessing the gymnasts' publicly available competitive performance data. Event scores ranging from 0.00 to 10.00 were retrieved online post-season from https://roadtonationals.com/results/standings/ for gymnasts who completed the questionnaires and who performed in a sufficient number of competitions throughout the season to have an NQS on at least one event.

\section{Statistical Analyses}

For the pilot study, data cleaning and preliminary analyses were conducted and reviewed to determine whether necessary assumptions for the substantive analyses were met. Missing values across the mindfulness and perfectionism items were assessed using Little's MCAR test $(p=.769)$, and no single item exceeded 1.3\% missing data. Due to the low number and random nature of missing values, Expectation-Maximization (EM) procedures were then used to impute missing values. As expected, the gymnastics performance data on each of the four events were 
negatively skewed. Reflection and log base 10 transformation approaches improved the distribution of scores for use in later parametric statistical analyses. For the current analytic sample, descriptive statistics were calculated on demographic and key study variables. Internal consistency reliabilities were also assessed for the measures, as were correlational analyses for the performance data and each of the subscales in the mindfulness and perfectionism instruments.

For the current study, cluster analytic approaches were conducted to establish whether unique profiles of mindfulness and perfectionism were present among the gymnast participants. Gymnasts' scores on the three mindfulness and two perfectionism subscales were submitted to cluster analysis in a two-step procedure: (1) Ward's hierarchical cluster analysis with squared Euclidean distance was conducted to help determine an initial number of clusters present among the participants, and (2) $k$-means iterative cluster analysis was used to further refine the cluster solution suggested by the initial hierarchical cluster analysis. The use of both hierarchical and iterative approaches to cluster analysis is supported in the literature (e.g., Gucciardi et al., 2012; Hair, Anderson, Tatham, \& Black, 1998; Kee \& Wang, 2008). A series of chi square analyses were then carried out to assess the number of athletes in each cluster who performed on each gymnastics event. This step allowed the research team to determine whether a sufficient sample size for each cluster/profile was met prior to conducting subsequent analyses. One-way analysis of variance (ANOVA) was then used to assess differences among the resulting mindfulness/perfectionism clusters in an objective measure of gymnastics performance (i.e., NQS) on each competitive event. Thus, four one-way ANOVAs were conducted to examine competitive performance differences across the clusters on each gymnastics event (i.e., vault, uneven bars, balance beam, floor exercise). Effect size estimates are reported as partial eta- 
squared for the ANOVA models, and as Cohen's $d$ for pairwise mean comparisons. All statistical analyses were computed in recent versions of SPSS.

\section{Results}

Of the total number of participants who completed the mindfulness and perfectionism survey measures $(N=301), 244$ gymnasts provided their name. It was only possible to access competition results for athletes who provided their names. Thus, the analytic sample for the current study was 244 gymnasts.

\section{Determining the Analytic Sample}

Prior to conducting the substantive analyses for the study, the research team conducted a series of preliminary analyses to assess potential differences between participants who would be retained in the analytic sample $(n=244)$ and those who would be omitted from the sample $(n=$ 57) on demographic and key study variables. T-tests indicated no statistically significant ( $p>$ $.05)$ differences between the gymnasts on the three mindfulness subscales. Statistically significant $(p<.05)$ differences were found, however, between the participants on the two perfectionism subscales. Gymnasts who did not report their name indicated slightly higher perfectionism scores than those who did report their name for both concern over mistakes (no name: $M=3.18, S D=0.78$; name: $M=2.87, S D=0.82$ ) and personal standards (no name: $M=$ 3.88, $S D=0.55$; name: $M=3.61, S D=0.58$ ) subscales. Gymnasts who did not report their name were also slightly higher in skill level than the larger group of participants who did provide their name (mean difference $=0.22$ ).

Given these differences, cluster analyses were conducted for both the full $(N=301)$ and analytic $(N=244)$ samples. Chi square analyses were then assessed to determine the proportion of athletes in each cluster who did and did not report their name. Although gymnasts who did not 
provide their name were disproportionately found in clusters high in perfectionism dimensions, parallel to the findings of the t-tests, the resulting cluster solutions were similar between the two samples, indicating that the gymnasts who omitted their name were likely not driving the cluster solution. The research team thus decided to move forward in reporting the cluster solution established with the analytic sample of gymnasts who provided their name $(N=244)$, and who would be included in the subsequent analyses incorporating performance data.

\section{Descriptive Statistics on the Analytic Sample}

Demographic information for the analytic sample of collegiate gymnasts is presented in Table 1 . Gymnasts in the present study ranged in age from 18 to 22 years $(M=19.46, S D=1.22)$ and were predominantly white $(n=196)$. A majority of the gymnasts reached level 10 prior to college $(n=204)$, had previous experience with a sport psychology professional $(n=162)$ and were satisfied with their experience $(n=130)$, and came into the study with no prior experience with mindfulness $(n=150)$.

Correlations, descriptive statistics, and internal consistency coefficients for the mindfulness and perfectionism subscale scores are outlined in Table 2. As expected, statistically significant $(p<.01)$ positive correlations arose among the three mindfulness subscales, and between the two perfectionism subscales. Significant, weak positive correlations were also found between personal standards perfectionism and both present moment attention $(r=.28)$ and awareness $(r=.16)$; yet there was no relationship between personal standards and acceptance $(r$ $<.01)$. Significant negative correlations arose between concern over mistakes perfectionism and each of the mindfulness subscales, though only one was moderate in size: present moment attention $(r=-.14)$, awareness $(r=-.15)$, and acceptance $(r=-.42)$. The negative relationships between mindfulness dimensions and concern over mistakes perfectionism, as well as the 
positive relationships between aspects of mindfulness and personal standards perfectionism, align well with previous research and theoretical perspectives of the constructs.

\section{Cluster Interpretation}

The five subscales were entered first into Ward's hierarchical cluster analysis. Based on visual inspection of the resulting dendogram and graphed coefficients from the agglomeration schedule, a three- or five-cluster solution appeared to provide the best description of the data. Together, the dendogram and agglomeration schedule help indicate points at which dissimilar clusters were being forced to merge, and thus provide information to determine relatively distinct groupings of individuals. Centroid values from both the three- and five-cluster solutions were then taken forward to be used as initial seed points in the $k$-means iterative cluster analyses.

The hierarchical and iterative cluster analytic approaches were then compared for both the three- and five-cluster solutions to assess the stability of the two solutions. Through crosstabulation, the percentage of cases similarly assigned to each cluster across the two analytic approaches could be assessed. Specifically, case classification for both the Ward's and $k$-means analyses indicated $69 \%$ similarity for the three-cluster solution, compared to a slightly improved $73 \%$ similarity for the five-cluster solution. Despite a small increase in stability for the fivecluster solution, power for subsequent statistical analyses would decrease notably given the smaller number of athletes in each group when moving from three- to five-clusters. The threecluster solution was therefore retained as it provided a nice explanation of gymnast mindfulness and perfectionism characteristics while permitting heightened power for subsequent analyses. Cluster means, standard deviations, and standardized scores for the final cluster solution are shown in Table 3. A visual representation of the final cluster solution is depicted in Figure 1. 
Interpretation of the subscale means for gymnasts in each cluster revealed the presence of three distinct profiles of mindfulness and perfectionism tendencies. The first cluster consisted of 87 athletes (35.7\%) with a moderate mindfulness and high perfectionism profile. The second cluster consisted of 71 athletes $(29.1 \%)$ with a low mindfulness and low/moderate perfectionism profile. The third cluster consisted of 86 athletes (35.3\%) with a high mindfulness and very low perfectionism profile. A series of four crosstabulation analyses were conducted to preliminarily assess the number of athletes in each profile who obtained an NQS on each competitive event during the season. Across the analyses, clusters ranged in size from 17 to 34 gymnasts. The group sizes were thus considered adequate for conducting subsequent one-way ANOVAs to assess potential performance differences across the three mindfulness and perfectionism profiles.

\section{Comparing Performance Across Clusters}

Prior to conducting parametric statistics using the performance data, transformations were made to improve the normality of the data. A series of four one-way ANOVAs were then computed using the transformed performance data to assess differences among the profiles. Event means, standard deviations, and sample sizes for each cluster are shown in Table 4. Results of the ANOVAs may be found in Table 5. No statistically significant $(\mathrm{p}<.05)$ differences in performance were found among the mindfulness and perfectionism profiles on the four competitive events. Effect sizes $\left(\eta_{p}^{2}\right)$ across the four analyses ranged from 0.03 to 0.06 , and power estimates for this set of analyses was low.

Exploratory post hoc t-tests were then conducted between groups with the greatest mean score differences on each event. The event NQS means compared, as well as the Cohen's $d$ effect size for each pairwise comparison, are indicated in Table 4. Significant mean score differences were found between clusters on vault and bars. On vault, the high mindfulness, very low 
perfectionism cluster performed significantly better than the low mindfulness, low/moderate perfectionism cluster. On bars, the moderate mindfulness, high perfectionism cluster performed significantly better than the high mindfulness, very low perfectionism cluster. Small to moderate effect sizes were found for each of the NQS comparisons across the four events. Statistically significant correlations also arose between vault performance and acceptance $(r=.24, p<.05)$, between bars performance and both personal standards $(r=.31, p<.01)$ and concern over mistakes $(r=.23, p=.05)$, between beam performance and personal standards $(r=.23, p=.05)$, and between floor performance and awareness $(r=.24, p<.05)$. Thus, despite the lack of significant findings when performance differences among the three profiles were considered together, potential relationships may exist between the mindfulness and perfectionism tendencies and competitive gymnastics performance.

\section{Discussion}

Three distinct profiles of mindfulness and perfectionism were observed among the gymnasts. Previous researchers have contributed to our understanding of how athletes may be grouped on each of these constructs independently (e.g., Gucciardi et al., 2012; Kee \& Wang, 2008); however, this is the first study to our knowledge that used a cluster analytic approach to understand how athletes in a judged sport experience mindfulness and perfectionism together. Previously, researchers have supported stable three- and four- cluster solutions for perfectionism and mindfulness, respectively. Thus, arriving at a three-cluster solution that helps to explain gymnasts' propensities for perfectionism and mindfulness concurrently aligns closely with existing notions of the constructs.

Furthermore, the current finding that gymnasts were classified quite evenly across three profiles indicates that mindfulness and perfectionism may be experienced in varied ways even 
within a relatively homogeneous group of elite athletes performing at a very high level. This gymnast-specific finding appears consistent with previous multi-sport research, as profiles of mindfulness and perfectionism tendencies have independently been found to vary markedly among high level athletes representing a broad range of sports (e.g., Gucciardi et al., 2012; Kee \& Wang, 2008). Although exploring a diverse set of personality characteristics was beyond the scope of the present study, these findings could be used to support the idea that there may not be just one adaptive personality profile for attaining an elite level of gymnastics. Furthermore, a national qualifying score (i.e., successful and consistent performance across a season) was achieved by athletes with and without notable levels of perfectionism and mindfulness.

When discussing differences observed across the mindfulness and perfectionism profiles, for instance gymnasts "high in mindfulness" or "low in perfectionism," scores are considered relative to the other gymnasts who participated in the study rather than compared to some normative criteria. Still, a basic understanding of how the current participants compared on the measurements to athletes included in previous research can provide clarity and highlight points of similarity and difference across studies. Zhang et al. (2017) found during development of the AMQ that subscale means among team and individual sport athletes ranged from 3.54 to 3.73 , with standard deviations in the 0.82 to 0.99 range. Comparatively, the gymnasts in our sample had slightly higher mindfulness scores overall, with means ranging from 3.63 to 4.11 across the three subscales, and lower standard deviations in the range of 0.48 to 0.59 . Taken together, the single-sport sample of gymnasts who participated in the current study reported slightly higher mindfulness tendencies with lower variation across responses than the multi-sport sample of athletes used when developing the instrument. 
In their research examining the validity of the Sport-MPS-2, Gotwals and Dunn (2009) reported mean scores for intercollegiate team sport athletes on the personal standards and concern over mistakes subscales were $3.68(S D=0.52)$ and $2.87(S D=0.68)$, respectively. In the present sample, the perfectionism scores among the intercollegiate gymnasts were equivalent or just slightly lower, with means of $3.61(S D=0.58)$ and $2.87(S D=0.82)$ for the personal standards and concern over mistakes subscales, respectively. Previously, Dunn et al. (2006) examined perfectionism among a sample of female figure skaters, a group slightly younger than the athletes in our gymnast sample but in a similar individual, judged sport context. The researchers found that the figure skaters' scores for personal standards and concern over mistakes were also lower than in the team sport sample, with subscale means of $3.33(S D=0.86)$ and $2.37(S D=0.97)$ for personal standards and concern over mistakes, respectively. Thus, the notion that certain sports may be considered more "perfectionistic" than others may not necessarily equate to higher individual reports of perfectionism tendencies. Perhaps athletes in such sport contexts have normalized the pursuit of perfection in a different manner than athletes in team sports might, and thus may self-report their own perfectionism relative to a higher standard resulting in lower scores. In any case, the gymnasts in the present study scored on the perfectionism dimensions in ways that align closely with intercollegiate athletes in various team sports studied previously.

Another primary aim of the current study was to assess potential performance differences across the mindfulness and perfectionism clusters. When gymnastics performance was considered on each of the four events across the three profiles, no significant differences were observed. It is important to note that the performance metric selected for comparison was relatively restricted in range given the calculation of the NQS as a snapshot of the better 
performances for each gymnast during the competition season (i.e., best and worst performances are dropped). Still, the NQS was selected intentionally as it is a practical performance metric used in collegiate gymnastics for qualification/placement in post-season competition. Given the utility of this performance score in the sport of gymnastics, the current findings may have practical significance. Specifically, high level performance appears to be attainable for athletes across competitive events, regardless of their propensities for mindfulness and perfectionism. Gymnasts may learn coping strategies that allow them to perform successfully with varying degrees of mindfulness and perfectionism tendencies. This finding may have practical utility for coaches and athletes - that individuals with different personality characteristics are all capable of high quality performance.

Despite the lack of statistically significant differences among the three profiles, noteworthy patterns in mean differences in event performance were observed. Researchers have argued that reliance on $p$ values when determining the meaning of results may be problematic, as the statistic is highly contingent on sample size and says relatively little about the real-world meaning of findings (e.g., Gigerenzer, 2004; Kruschke, 2013; Wilkinson, 2014). In recent years, researchers have recommended interpreting test statistics through a contextual lens to better understand the practical significance of the results (Andersen, McCullagh, \& Wilson, 2007). A similar process was recently adopted in a study of mindfulness-based programming for injury prevention (Ivarsson, Johnson, Andersen, Fallby, \& Altemyr, 2015), and we have followed these recommendations. In the context of gymnastics, very small differences in performance scores may mean the difference between placing and not placing, between qualifying to post-season competition and not qualifying. 
For example, the difference in NQS for the top 10 athletes on each competitive event during the 2019 regular season was approximately five hundredths of a point, 0.05 . The mean score differences in the one to two tenth of a point range, 0.1 to 0.2 , observed between profiles established in the current study were therefore considered to have real-world meaning in the context of gymnastics. For the comparison across profiles, $\eta_{p}^{2}$ effect size values ranged from 0.03 to 0.06 , indicating that approximately three to six percent of variance in event performance may be accounted for by profile membership. In our sample of gymnasts, NQS values had a range of approximately one point on each event. Given the explained variance in event performance we observed in the study, a three to six percent change in NQS may mean the difference between first and tenth in the nation. Additionally, when effect sizes were computed for event scores with maximum variation between profiles, Cohen's $d$ values ranged from 0.37 to 0.65 indicating the presence of some moderate effect sizes. Thus, it is possible that some qualities of mindfulness and perfectionism serve athletes in more adaptive ways on certain competitive events.

In support of this finding, statistically significant mean differences arose between the profiles compared on vault and on bars in post hoc pairwise comparisons. On vault, gymnasts highest in mindfulness performed best; whereas, on bars, gymnasts highest in perfectionism performed best. When observing the mean score patterns on each event across profiles, gymnasts highest in perfectionism performed relatively better than gymnasts in profiles lower in perfectionism on bars, beam, and floor. These trends suggest that the degree of mindfulness and perfectionism qualities most favorable for performance may vary by event, and that in contrast to existing research (e.g., Hill et al., 2018) perfectionism may be adaptive for competitive gymnastics performance in the context of the study. 
It seems possible that the mindfulness and perfectionism qualities favorable for performance may also differ from those favorable for wellbeing. For instance, although the findings of the current study lend initial, tentative support for the physical performance benefits of perfectionism in gymnastics, high levels of concern over mistakes perfectionism has previously been considered maladaptive for the emotion and wellbeing of athletes (e.g., Hill et al., 2018). Researchers have provided some evidence of the potential benefits of mindfulness for the wellbeing of athletes, including reduced competitive anxiety, stress, and burnout; injury prevention; and increased confidence and self-efficacy (e.g., Noetel et al., 2017). Although the question of athlete wellbeing was not within the scope of the current research, future researchers might consider including such assessments when studying these constructs to better understand both the quality of athletes' experiences alongside their objective performance.

The current findings may also have practical implications for researchers and practitioners interested in delivering mindfulness-based interventions to athletes such as those who participated in our study. In light of some equivocal research findings in the mindfulness literature, researchers have begun to question the efficacy of mindfulness-based interventions for all participants, and have raised the potential for adverse effects of meditation-related experiences (Van Dam et al., 2018). Understanding individual characteristics that may predispose individuals to have more favorable versus adverse effects to meditation-related practices may help to direct our research and applied efforts toward participants who may benefit most from them. For instance, concern over mistakes perfectionism has been positively associated with anxiety and depressive symptoms (Hill et al., 2018) - experiences that align with the relatively rare albeit real meditation-related adverse effects that have been observed (Van Dam et al., 2018). Future researchers might aim to understand if differences exist across profiles 
of individual characteristics in athletes' readiness or desire to engage in mindfulness-based interventions, or in the effectiveness of such interventions among distinct profiles. By assessing these constructs prior to interventions, researchers or practitioners might then have a clearer understanding of who may benefit most from mindfulness-based interventions rather than assuming that all athletes participating have the capacity to benefit equally from such practices.

We selected the NQS as our performance measure in the current research based on its practical use in determining placement in post-season competition. We recognize, however, that there may be potential limitations to this metric in our study. For instance, given the way the NQS is calculated, only a portion of a gymnasts' performances throughout the season may be taken into consideration. Further, that portion of the season that is calculated into the NQS would not account for poorer competitive performances if gymnasts competed in most meets during the season. This performance measure may therefore be overly reductive and restricted in range, potentially limiting our ability to notice performance differences across the profiles on the different apparatus using inferential statistics. Future researchers might consider using a different performance metric (e.g., true average of all performances or range of performance scores) or a mix of measures to study the relationship between these concepts and performance.

Self-report measures were used to assess individual experiences of both mindfulness and perfectionism among the gymnasts. When self-report measures are involved, there is a potential for bias in the form of accurate recall or social desirability. The potential for social desirability bias in the reported experiences may be especially salient given that athletes were asked to provide identifying information. During the initial screening analyses, small but significant differences in perfectionism scores were noted between gymnasts who did and did not provide their name, with those who did not report their name scoring higher in dimensions of 
perfectionism than those who did. Although provision of names was a necessary component for carrying out the current study with objective performance measures, it should be noted that authentic self-report of perfectionism may be influenced to some extent when identifying information is provided.

We accessed a large sample of elite level gymnasts to participate in the current study. Still, only small effects were found across profiles relative to objective performance - likely due to the restricted range in performance scores observed among this relatively homogeneous, highlevel sample of athletes, and to the inherently small increments of change frequently observed in the scoring system within gymnastics. With a larger sample of athletes, future researchers may be able to heighten the power to detect small, meaningful differences in performance among the different profiles. Still, that differences in performance outcomes were found between profiles may hold meaning for coaches, athletes, and practitioners operating in a sport where exceedingly small differences in scores often can have large practical consequences.

Several directions for future research stem from the present findings. In the current study, we aimed to take a nuanced approach to studying the relationship between mindfulness and performance among collegiate gymnasts by also considering perfectionism as a contextually salient factor in the sport. We found individual profiles of mindfulness and perfectionism, and assessed how those quantitative reports were associated with objective measures of gymnastics performance. Still, further nuance in the contextually situated experiences of gymnasts seems attainable. Researchers have previously acknowledged the importance of understanding impact mechanisms of various facets of mindfulness (Birrer, Röthlin, \& Morgan, 2012). By taking a mixed method approach, future researchers might better understand not only athlete profiles of 
mindfulness and perfectionism, but also perspectives regarding which aspects of an athlete's individual profile are perceived to have the greatest impact on performance outcomes.

In keeping with the recommendation to assess determinants of both performance and wellbeing, qualitative or mixed method approaches may also be used to more deeply understand gymnasts' lived experiences regarding the factors that impact both quality of performance and quality of experience. Through in-depth interviews, perhaps complemented by measures to assess personal wellbeing, researchers might gain a more holistic perspective regarding the facets of mindfulness and perfectionism that impact athletes' physical and mental health. Such an approach would be congruent with the whole-athlete wellness perspective currently being emphasized through the adoption of mental health practitioners in college and university athletic departments across the nation.

On a final note, the present study examined gymnasts' typical experiences of mindfulness and perfectionism in their sport setting. A potential next step might then be to facilitate a mindfulness- or meditation-based intervention for athletes to enhance skills in this domain prior to exploring links to performance and wellbeing. Because scholars have recently noted the potential for individuals to experience meditation-related adverse effects (Van Dam et al., 2018), an understanding of individuals' readiness or openness to engage in such an intervention might help us to reach those athletes who may benefit most. Future researchers might therefore consider including a survey item or interview question to assess athletes' openness to engaging in mindfulness-based practices in the process of this type of research to aid in future screening of personality characteristics/indicators that may be associated with or contraindicated for readiness to participate in an intervention. This step may aid in minimizing risk and maximizing benefit of future mindfulness-based research efforts. 
We are cautiously optimistic about the findings presented herein, bolstered by recommendations that real-world meaning can be gained from interpreting findings based on the study context (e.g., Andersen et al., 2007). Regarding mindfulness and perfectionism tendencies, three distinct profiles were found among collegiate gymnasts performing at high levels for their respective institutions, suggesting that more than one profile of characteristics may be adaptive for reaching high levels of performance in gymnastics. Although performance differences were not found among the profiles when analyzed together, small to moderate effect sizes were observed across the four events when profiles with maximum variation were compared. These results are promising, and may provide initial support for the qualities that may be most adaptive for performance on each competitive event. 


\section{Tables and Figures}

Table 1

Descriptive Statistics of Demographic Variables for Intercollegiate Gymnasts $(N=244)$.

\begin{tabular}{|c|c|c|c|c|c|}
\hline Variables & $\begin{array}{c}\text { Valid } \\
\%\end{array}$ & $n$ & $N$ & $M$ & $S D$ \\
\hline Age & & & 243 & 19.46 & 1.22 \\
\hline Highest Level of Gymnastics & & & 244 & & \\
\hline 9 & 12 & 28 & & & \\
\hline 10 & 84 & 204 & & & \\
\hline Elite & 4 & 9 & & & \\
\hline Other & 1 & 3 & & & \\
\hline Race & & & 242 & & \\
\hline White & 81 & 196 & & & \\
\hline Black or African American & 5 & 13 & & & \\
\hline Asian & 5 & 12 & & & \\
\hline American Indian, Alaska Native, Pacific Islander & 3 & 6 & & & \\
\hline From multiple or other races & 6 & 15 & & & \\
\hline Previous Experience with a SPP & & & 243 & & \\
\hline Both Individually and in a Team Setting & 31 & 75 & & & \\
\hline Individually & 8 & 20 & & & \\
\hline In a Team Setting & 28 & 67 & & & \\
\hline No Previous Experience & 33 & 81 & & & \\
\hline Satisfaction with a SPP & & & 170 & & \\
\hline Extremely/Somewhat Satisfied & 76 & 130 & & & \\
\hline Neither Satisfied nor Dissatisfied & 19 & 33 & & & \\
\hline Extremely/Somewhat Dissatisfied & 4 & 7 & & & \\
\hline Previous Experience with Mindfulness & & & 238 & & \\
\hline Previous Experience & 37 & 88 & & & \\
\hline No Previous Experience & 63 & 150 & & & \\
\hline
\end{tabular}

Note. SPP = sport psychology professional. Valid \% values have been rounded to the nearest whole number. 
Table 2

Zero-Order Correlations, Descriptive Statistics, and Internal Consistency Coefficients of Mindfulness and Perfectionism Subscale Scores $(N=244)$.

\begin{tabular}{lcccccccc}
\hline Variables & 1 & 2 & 3 & 4 & 5 & $M$ & $S D$ & $\alpha$ \\
\hline 1. Present Moment Attention & -- & $.61^{* *}$ & $.59^{* *}$ & $.28^{* *}$ & $-.14^{*}$ & 4.00 & 0.49 & 0.75 \\
2. Awareness & & -- & $.46^{* *}$ & $.16^{*}$ & $-.15^{*}$ & 4.11 & 0.48 & 0.77 \\
3. Acceptance & & -- & $<.01$ & $-.42^{* *}$ & 3.63 & 0.59 & 0.75 \\
4. Personal Standards & & & -- & $.47^{* *}$ & 3.61 & 0.58 & 0.77 \\
5. Concern over Mistakes & & & - & & -- & 2.87 & 0.82 & 0.88
\end{tabular}

$* * p<.01, * p<.05$

Table 3

Cluster means, standard deviations, and z scores for the three-cluster solution $(N=244)$.

\begin{tabular}{|c|c|c|c|c|c|c|c|c|c|}
\hline \multirow[t]{2}{*}{ Variables } & \multicolumn{3}{|c|}{$\begin{array}{c}\text { Cluster } 1 \\
\text { (Moderate mindfulness, } \\
\text { high perfectionism) } \\
(n=87)\end{array}$} & \multicolumn{3}{|c|}{$\begin{array}{c}\text { Cluster } 2 \\
\text { (Low mindfulness, } \\
\text { low/moderate perfectionism) } \\
(n=71)\end{array}$} & \multicolumn{3}{|c|}{$\begin{array}{c}\text { Cluster } 3 \\
\text { (High mindfulness, } \\
\text { very low perfectionism) } \\
\qquad(n=86)\end{array}$} \\
\hline & $M$ & $S D$ & $z$ & $M$ & $S D$ & $z$ & $M$ & $S D$ & $z$ \\
\hline $\begin{array}{l}\text { 1. Present Moment } \\
\text { Attention }\end{array}$ & 4.10 & .39 & .21 & 3.54 & .40 & -.92 & 4.27 & .38 & .55 \\
\hline 2. Awareness & 4.20 & .44 & .19 & 3.72 & .36 & -.83 & 4.35 & .39 & .50 \\
\hline 3. Acceptance & 3.51 & .50 & -.20 & 3.19 & .44 & -.75 & 4.11 & .41 & .82 \\
\hline $\begin{array}{l}\text { 4. Personal } \\
\text { Standards }\end{array}$ & 4.02 & .43 & .72 & 3.28 & .46 & -.56 & 3.45 & .56 & -.26 \\
\hline $\begin{array}{l}\text { 5. Concern over } \\
\text { Mistakes }\end{array}$ & 3.63 & .50 & .93 & 2.87 & .59 & .01 & 2.09 & .44 & -.94 \\
\hline
\end{tabular}


Figure 1

Graphed Cluster Solution of Standardized Mindfulness and Perfectionism Subscale Scores $(N=$ 244).

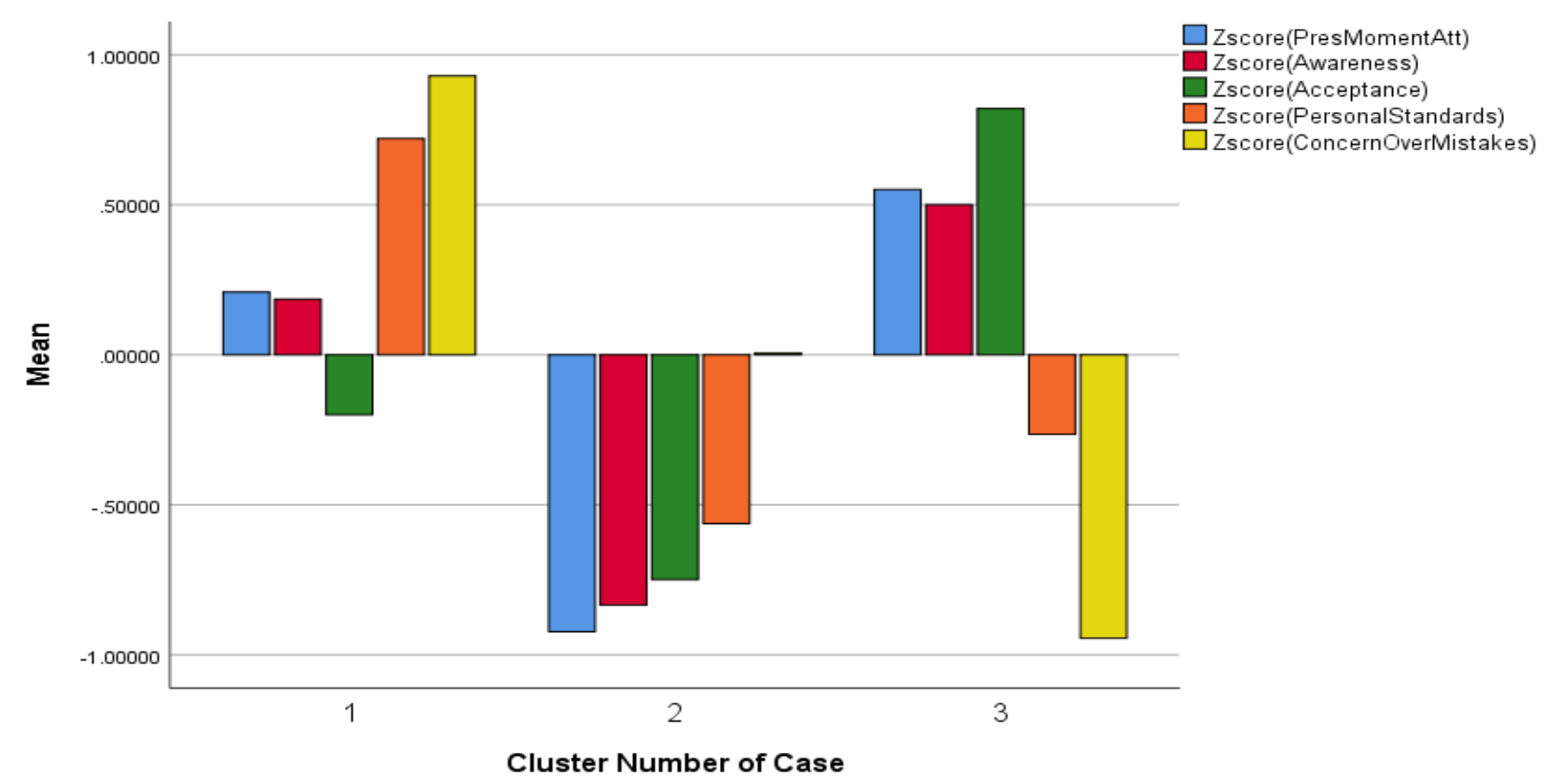

Table 4

Event means, standard deviations, and sample sizes for the three-cluster solution.

\begin{tabular}{lcccccccccc}
\hline & $\begin{array}{c}\text { Cluster 1 } \\
\text { (Moderate mindfulness, } \\
\text { high perfectionism) }\end{array}$ & $\begin{array}{c}\text { Cluster 2 } \\
\text { (Low mindfulness, } \\
\text { low/moderate } \\
\text { perfectionism) }\end{array}$ & $\begin{array}{c}\text { Cluster 3 } \\
\text { (High mindfulness, } \\
\text { very low perfectionism) }\end{array}$ & $\begin{array}{c}\text { Effect } \\
\text { Size }\end{array}$ \\
\cline { 2 - 11 } & $M$ & $S D$ & $n$ & $M$ & $S D$ & $n$ & $M$ & $S D$ & $n$ & $d$ \\
\hline 1. Vault NQS & 9.63 & .25 & 34 & $9.55^{*}$ & .23 & 18 & $9.69^{*}$ & .18 & 30 & -0.65 \\
2. Bars NQS & $9.64^{*}$ & .28 & 32 & 9.50 & .37 & 17 & $9.44^{*}$ & .41 & 25 & 0.57 \\
3. Beam NQS & $9.66^{*}$ & .24 & 30 & 9.61 & .22 & 17 & $9.56^{*}$ & .31 & 27 & 0.37 \\
4. Floor NQS & $9.72^{*}$ & .17 & 31 & $9.60^{*}$ & .26 & 18 & 9.65 & .26 & 28 & 0.53 \\
\hline
\end{tabular}

Note. NQS = National Qualifying Score. * Indicates event means compared in effect size calculations. 
Table 5

Univariate Analysis of Variance Exploring Mindfulness and Perfectionism Profile Differences in Competitive Performance on Each Event.

\begin{tabular}{lcccc}
\hline & $d f$ & $F$ & $p$ & $\eta_{p}^{2}$ \\
\hline 1. Vault & 2 & 2.07 & .13 & .05 \\
2. Bars & 2 & 2.31 & .11 & .06 \\
3. Beam & 2 & 1.08 & .35 & .03 \\
4. Floor & 2 & 1.10 & .34 & .03 \\
\hline
\end{tabular}




\section{Literature Review}

In the field of sport psychology, mindfulness literature has expanded greatly in recent years (e.g., Noetel, Ciarrochi, Van Zanden, \& Lonsdale, 2017). With this growth, researchers have begun to consider new relationships between mindfulness and existing psychological constructs in the context of sport. Throughout the present literature review, extant research related to mindfulness, perfectionism, and performance in sport will be discussed. Specifically, early sections will provide an overview of historical roots and approaches to mindfulness. Following this background context, applications of mindfulness in sport, and relationships between mindfulness, athletic performance, and other psychological aspects of sport will then be addressed. Perfectionism, a construct found to be related to both mindfulness and athletic performance, will also be explored. Given the relevance of measurement to the study of key constructs in sport and performance psychology, measurement of mindfulness, perfectionism, and performance will be focal elements of the review. Directions for future research that draw from and extend existing empirical literature will then be offered, and a conceptual model integrating mindfulness, perfectionism, and performance will be outlined to provide a visual representation of potential relationships among the constructs discussed throughout the review.

\section{Mindfulness: A Brief Backdrop}

The current section will provide a brief history of mindfulness, its introduction into Western culture and professional practice, and mindfulness approaches introduced within the initial third wave of cognitive-behavioral therapies. Such a backdrop is intended to provide context from which to better understand the subsequent advances made regarding mindfulness applications and research evidence. 


\section{Historical Roots}

Mindfulness is an Eastern philosophical concept rooted in ancient Hindu and Buddhist meditation practices that appears in various forms within Islamic, Christian, and Judaic traditions (Andersen \& Waterson, 2017). Only since the 1970s, however, have mindfulness-related influences emerged and become integrated into Western culture and psychology. As mindfulness developed within North American research and practice, two different conceptualizations of the mindfulness construct were developed (Pineau, Glass, \& Kaufman, 2014). Langer (2000) developed a concept of mindfulness that involves "a flexible state of mind in which we are actively engaged in the present, noticing new things and sensitive to context" (p. 220). The defining features of this conceptualization of mindfulness center around noticing one's context and in so doing enhancing one's ability to effectively respond to aspects of that context. Another primary conceptualization of mindfulness, developed by Kabat-Zinn (1990, 1994), was more closely rooted in ancient Buddhist philosophy. Among the many definitions that have been brought forth among scholars and practitioners, Kabat-Zinn's (1994) description of mindfulness has perhaps been most widely used: "paying attention in a particular way: on purpose, in the present moment, and nonjudgmentally" (p. 4). In alignment with this Eastern conceptualization, several core concepts of mindfulness have arisen in the literature, including present-focused awareness, an accepting or open attitude, a nonjudgmental approach, and compassion for self and others (Zizzi, 2017). These elements capture much of the essence of the concept and practice of mindfulness, and are foundational to many of the more recently developed approaches to promoting physical and psychological wellbeing (e.g., Hayes, Strosahl, \& Wilson, 1999) and enhancing sport performance (e.g., Kaufman, Glass, \& Pineau, 2017). 
Mindfulness was originally introduced into Western practices within the field of mental health in the form of third wave cognitive-behavioral therapy treatment programs for depression, anxiety, chronic pain, and post-traumatic stress disorder (e.g., Kabat-Zinn, 1982; Teasdale et al., 2000), and has since expanded into a broad range of physical and mental health fields. Teachings grounded in the philosophical concept of mindfulness have been used in both clinical and nonclinical settings. Such teachings have been integrated into approaches including mindfulnessbased stress reduction (MBSR; Kabat-Zinn, 1982), dialectical behavior therapy (DBT; Linehan, 1993), mindfulness-based cognitive therapy (MBCT; Segal, Williams, \& Teasdale, 2002), and acceptance and commitment therapy (ACT; Hayes et al., 1999). Put quite broadly, these programs and approaches have systematically assisted individuals and/or groups in developing mindful awareness as a self-regulatory approach to better manage physical, psychological, and psychosomatic concerns, or to alter the relationship one has with such concerns. The efficacy of mindfulness and acceptance-based programs has been demonstrated across a broad range of presenting concerns, and meta-analyses (McAlarnen \& Longshore, 2017) in the mindfulness literature have examined outcomes including stress, anxiety, depression, wellness, performance, communication/interpersonal relationships, and outcomes among healthcare practitioners that support mindfulness-based approaches as having utility.

\section{Mindfulness-Based Approaches}

Often regarded as third wave cognitive-behavioral therapies, a series of mindfulnessbased approaches were developed to assist people in cultivating awareness and acceptance to more effectively cope with a range of psychophysiological concerns. MBSR (Kabat-Zinn, 1982) is often considered the first structured program that brought Eastern mindfulness meditation philosophies into Western medical and psychotherapy practices. The program systematically 
assists individuals in developing/enhancing mindful attention/awareness as a self-regulatory approach for individuals to better manage and cope with clinically-based physical, psychosomatic, and psychiatric concerns.

In its wake, MBCT (Segal et al., 2002) was established as a brief group intervention to help prevent the recurrence of major depression (Dimidjian, Kleiber, \& Segal, 2009). The MBCT approach employs mindfulness meditation practices, depression psychoeducation, and cognitivebehavioral strategies. ACT (Hayes et al., 1999) as a mindfulness and acceptance-based therapy was designed to help individuals reduce unnecessary distress and move toward developing values-driven behaviors (Batten, 2011). The ACT practitioner and client work together using the following six processes to help improve client functioning and well-being, and to enhance psychological flexibility: acceptance, contact with the present moment, defusion, values, self-ascontext, and committed action. The aim of ACT is not to directly change psychological processes, but rather to change the relationship one has with those psychological processes through an integration of the six key areas of focus in ACT (Batten, 2011). Empirically-based findings have supported the efficacy of such third wave therapies within general and clinical populations on outcomes including anxiety, stress, and depression (McAlarnen \& Longshore, 2017). As mindfulness and acceptance-based approaches garnered support as efficacious therapies within different contexts, researchers and practitioners sought to develop domainspecific mindfulness approaches to working with clients.

\section{Mindfulness in Sport and Performance Psychology}

With increasing attention being paid to the benefits of mindfulness within health-related fields, scholars and practitioners in the field of sport and performance psychology soon recognized the potential value of these mindfulness teachings and their applicability to sport. 
Sport-specific mindfulness approaches to performance enhancement thus began to emerge, including the mindfulness-acceptance-commitment approach (MAC; Gardner \& Moore, 2004, 2007), mindful sport performance enhancement (MSPE; Kaufman et al., 2017), and mindfulness meditation training for sport (MMTS; Baltzell \& Summers, 2017). Although the approaches differ somewhat in their focus and delivery, they hold at their foundation the notion that our cognitive and emotional states do not necessarily need to be altered directly to promote positive behavioral and affective outcomes - a notion that differs quite notably from more traditional approaches in the field of sport psychology.

\section{Mindfulness-Based Approaches in Sport}

The MAC approach (Gardner \& Moore, 2004, 2007) brought together facets of acceptance- and mindfulness-based approaches by translating the ACT framework into the context of sport. The approach developed from the notion that our cognitions and emotions need not be directly altered to promote positive behavioral outcomes as has often been the focus of traditional cognitive-behavioral or psychological skills training approaches to enhancing sport performance. The MAC approach is therefore an alternative/supplemental approach to facilitating sport performance that helps athletes clarify values and develop mindful, nonjudgmental, present-moment acceptance of internal experiences. The MAC framework consists of seven modules covering the following topics: introductions to psychoeducation, mindfulness, cognitive defusion, and values; acceptance as an alternative to avoidance of unpleasant experiences; commitment to behaviors in alignment with values and performance goals; and consolidation/maintenance of mindfulness, acceptance, and commitment skills learned throughout the program. These modules may be completed across seven or more group or individual sessions. In a chapter review of current mindfulness and acceptance-based approaches 
with collegiate student-athletes, the MAC approach has been shown to be effective in a variety of outcomes, including enhancing concentration, experiential acceptance, mindful attention and awareness, and performance in sport (Wolanin \& Gross, 2016).

The MSPE program (Kaufman, Glass, \& Arnkoff, 2009) extends foundational mindfulness-based approaches to athletes. The approach differs from MAC in that MSPE does not focus on values or commitment. MSPE was developed as an integration of MBSR and MBCT, including key exercises (i.e., body scan, mindful breathing, mindful yoga, raisin exercise, sitting and walking meditation), and is suitable for groups. The program is typically six sessions of 90 minutes in duration over the course of about six weeks, including the following session foci: building mindfulness fundamentals, mindfully stretching limits of the body, strengthening attention and embracing reality, and learning to embody the mindful performer. Mindfulness within the MSPE framework is viewed as compatible with flow and peak performance theories in sport. Athletes' general satisfaction with sport performance, has been significantly higher post-MSPE workshop than pre-workshop, and overall state flow has been found to significantly change over the course of the MSPE training.

The MMTS approach (Baltzell \& Summers, 2017) was developed and introduced to athletes as mental training through meditation. Four main areas to the MMTS program include open awareness, concentration exercises, compassion for self and others, and acceptance of negative thoughts and emotions. The program consists of 12 training sessions, with two 30minute meetings per week, all of which integrate discussion regarding the application of mindfulness skills to both practice and competition settings. MMTS has been found to increase mindfulness, focus, and awareness among athletes.

\section{Linking Mindfulness-Based and Traditional PST Approaches}


Primary psychological skills training (PST) approaches within sport and performance psychology have traditionally included goal setting, imagery/visualization, arousal regulation, and self-talk among others. A key aim of PST approaches has often been to intentionally change or control the cognition, emotion, or affect of athletes, and to develop self-regulation skills to attain enhanced performance (Hardy, Jones, \& Gould, 1996; Meichenbaum, 1977). However, researchers have recently raised questions regarding the efficacy of traditional PST approaches for enhancing real-world athletic performance (Birrer, Röthlin, \& Morgan, 2012; Gardner \& Moore, 2006). Mindfulness and acceptance-based models are centered around the definitional elements of non-judgmental, moment-to-moment awareness and acceptance of our thoughts, feelings, and behaviors. Developing the ability to direct attention where desired without exerting intentional control over our internal experiences is thought to enhance athletes' mental efficiency, through greater awareness and acceptance of internal experience, rather than striving to devote deliberate, conscious control over internal experience (e.g., Gardner \& Moore, 2012; Thompson, Kaufman, De Petrillo, Glass, \& Arnkoff, 2011; Sappington \& Longshore, 2015). Thus, a central focus of mindfulness-based approaches to facilitating performance is to modify the relationship one has with internal experiences, rather than to actively change, control, or reduce such cognitions, emotions, and psychological sensations as many of the more traditional PST approaches encourage. In support of a mindful approach to facilitating sport performance, Bernier, Thienot, Codron, \& Fournier (2009) found that a greater number of athletes who took part in a mindfulness and acceptance-based program improved their national performance ranking than those who took part in a traditional PST program. Birrer et al. (2012) suggested attention, acceptance, clarity of values, self-regulation, non-attachment, less rumination, and flexibility as potential impact mechanisms of mindfulness for athletes. While both mindfulness 
and more traditional PST methods may have performance benefits as a common thread, the process or approach to moving closer to those desired performance benefits may look somewhat different through each theoretical view.

Although researchers have posited that mindfulness and traditional PST frameworks may have different theoretical roots (Gardner \& Moore, 2012; Sappington \& Longshore, 2015), perhaps the empirical literature can also point toward a more symbiotic relationship between these prominent approaches to performance enhancement in sport. Research linking mindfulness to the adoption of mental skills may help to conceptualize the relationship between mindfulness and traditional PST approaches. Kee and Wang (2008) used a cluster analytic approach to explore the relationship between mindfulness profiles of university athletes, and the adoption of traditional mental skills. The researchers found that the cluster of individuals highest in aspects of mindfulness showed the most frequent use of mental skills in sport (i.e., goal setting, self-talk, attentional and emotional control, and imagery). Additionally, a recent study exploring relationships between the use of a particular mental skill, self-talk, and real-world competitive performance among collegiate gymnasts provided support for the performance benefits of selftalk for athletic performance (Van Dyke, Van Raalte, Mullin, \& Brewer, 2018). The researchers further noted the important role that automaticity may play in effectively using self-talk while also conserving key cognitive and attentional resources, one of the noteworthy benefits of mindfulness-based approaches noted previously. Furthermore, Baltzell and Diehl (2017) outline through their Mindfulness-Flow-Performance Model in Sport that traditional PST approaches may strengthen the connection between the present-mind state and task-relevant attention characteristic of mindfulness, and the experience of flow in sport performance. 
Rather than mindfulness and traditional PST existing as distinct approaches to sport performance enhancement, perhaps instead mindfulness provides athletes with the awareness and openness to experience that allows them to benefit more from or be more inclined to integrate mental skills into their repertoire of practices. Little work has explored the idea of including mindfulness as a pre-cursor to subsequent interventions, or as a concurrent intervention. For example, it would be possible to provide a team with psychoeducational sessions using a PST format while including mindfulness-based exercises to individual athletes. In sum, a mindful approach to sport performance enhancement may serve the athlete via multiple pathways - by cultivating greater awareness and acceptance of internal experience which directly benefits performance, by cultivating a present-mind state to more openly integrate other adaptive mental skills which together may benefit performance, or perhaps by another pathway altogether. Further research pertaining to mindfulness and athletic performance could foster greater understanding regarding the influences of mindfulness in the context of sport.

\section{Mindfulness, Performance, and Psychological Aspects of Sport}

Empirical literature examining the influence of mindfulness-based teachings on sport performance and factors known to be closely linked to sport performance has grown abundantly within the past decade. Once such factor often studied alongside mindfulness and sport performance is flow. Flow states tend to arise when the skill level of the individual matches the challenge of the task, and are often described as psychological states of optimal experience characterized by feelings of enjoyment, engagement, and absorption, engagement in the present task (Csikszentmihalyi, 1990). Additionally, flow has been thought to share features in common with mindfulness, including sustained attention on the task or action at hand, and awareness of the present-moment experience (Baltzell \& Diehl, 2017). Furthermore, flow has been considered 
a relevant outcome variable of mindfulness-based interventions given the link between flow and high levels of performance (e.g., Sappington \& Longshore, 2015). Given the relevance of flow to the present discussion of mindfulness and performance, studies integrating flow within their methodologies will also be presented.

Illustrating growth in the literature on mindfulness in sport, Sappington and Longshore (2015) conducted a systematic review of 19 empirical studies spanning a variety of methodologies. The authors provided preliminary support for the use of mindfulness-based approaches to enhance athletic performance. Studies selected for inclusion in the review explored applied mindfulness interventions, and employed athlete samples. Outcomes of interest were all related either directly (e.g., objective and subjective measures of sport performance) or indirectly (e.g., competitive state anxiety and flow) to athletic performance. Results of the systematic review indicated that randomized trials supported improvements in objective performance measures, as well as outcomes related to enhanced performance including flow and competitive anxiety (further details regarding randomized trials will be outlined later in this section). Methodologies including case studies, qualitative studies, and nonrandomized trials substantiated these findings, indicating that mindfulness-based interventions may be promising for facilitating sport performance and improving factors associated with performance and wellbeing (e.g., anxiety, flow, psychological flexibility).

Specifically, reviewed case study, qualitative research, and nonrandomized trial findings (Sappington \& Longshore, 2015) offered support for the performance benefits of mindfulness interventions with individual athletes across a range of sports (e.g., lacrosse, diving, golf, soccer, swimming, figure skating, equestrian, long-distance running, and power lifting). Benefits associated with the practice of mindfulness included improved subjective and objective measures 
of performance, as well as improvements across measures related to performance (e.g., increased training intensity and focus; enhanced engagement in performance and experiences of flow; increased acceptance, nonjudgmental awareness, positive self-perceptions, self-confidence, and mindfulness skills; and decreased competitive anxiety, worry, and perfectionism). However, due to the noteworthy limitations in the methodological rigor and empirical quality of studies conducted to date, further research is needed to provide support for the efficacy of mindfulnessbased interventions in sport.

Drawing upon the findings discussed thus far, one might begin to develop a cautiously optimistic stance relative to the effectiveness of mindfulness for facilitating athletic performance. The state of empirical literature in the field of mindfulness and sport performance has been suggested to lack robustness, and still further researchers have aimed to synthesize the available information to arrive at a better understanding of the present state of evidence in mindfulness research. A recent overview of 36 meta-analyses and systematic reviews in the mindfulness literature has raised an encouraging yet somewhat tentative story regarding the performance enhancing effects of mindfulness-based interventions (McAlarnen \& Longshore, 2017). Among the selected meta-analyses and review articles, a variety of methodological designs arose (e.g., randomized control trials, nonrandomized trials, and observational studies), with a range of intervention formats (e.g., mixed mindfulness-based interventions, meditation, MBCT, and MBSR). Study outcomes were not solely based in sport-related factors, and included positive psychological outcomes (e.g., improved well-being, self-concept, and positive affect), physical health outcomes (e.g., improved sleep, reduced symptomatology), behavior change outcomes (e.g., weight management), and clinical psychological outcomes (e.g., relapse reduction). Of particular interest, the researchers analyzed how mindfulness-based interventions affected 
outcomes such as anxiety/stress, depression, and performance in clinical, nonclinical, and medical samples across multiple fields, including education, medicine, psychology, and sport. The reported effect sizes can be interpreted based on Cohen's $d$ magnitude conventions (small $=$ .20$, medium $=.50$, large $>.80)$.

McAlarnen and Longshore (2017) suggested that mindfulness-based interventions have been fairly consistent and robust in effectively reducing and managing depression, anxiety, and stress in nonclinical and clinical populations of adults. Outcomes like depression have been studied more extensively in clinical populations than in nonclinical and medical populations, however, suggesting that the strength of evidence may depend on the population of interest. Furthermore, there is relatively little meta-analytic evidence pertaining specifically to outcomes of anxiety, stress, and depression relative to mindfulness-based interventions among athlete and student populations. One must therefore be mindful when attempting to generalize such findings beyond the scope of the available evidence within a given population.

Surprisingly, given the spreading popularity of mindfulness as a method to facilitate performance, relatively few meta-analyses have examined the evidence linking mindfulnessbased interventions to performance across domains (McAlarnen \& Longshore, 2017). At the time of publication, just two meta-analyses included evidence pertaining to measures of objective performance, and those were in the domains of academic and cognitive performance among student populations. In addition, meta-analytic data on outcomes related to performance (e.g., attention, resilience, social competence, and positive emotions) were included. Three systematic reviews represented developing evidence pertaining to mindfulness-based interventions and performance in sport; however, effects of mindfulness-based interventions on athletic performance outcomes were not reported due to the gap in meta-analytic findings. The authors 
reported inconclusive evidence regarding the use of mindfulness-based interventions to enhance performance, due mainly to the limited degree of evidence available. Although the effect sizes for academic and cognitive performance ranged from medium to large, only small to medium effects were found for the performance-related outcomes. Thus, despite many anecdotal accounts and favorable preliminary findings sprinkled throughout the literature, experimental evidence has yet to confirm the link between mindfulness-based interventions and performance outcomes. In light of such findings, the authors have called for more literature using randomized controlled trials, increased sample sizes, and direct or objective measures of performance (McAlarnen \& Longshore, 2017). Such recommendations support future research efforts to contribute to the mindfulness literature through inclusion of more rigorous and varied study methodologies.

Noetel et al. (2017) systematically reviewed mindfulness and acceptance-based approaches to promoting key outcomes in sport (e.g., athletic performance, mindfulness, flow), with 66 total studies meeting criteria for inclusion. The studies selected included 21 studies with observational designs (e.g., correlational designs), and 43 studies involving an intervention (i.e., 17 randomized controlled trials, 14 non-randomized control group trials, and 12 trials without a control group). Athletes $\left(N=3,908, M_{\text {age }}=22.89\right.$ years $)$ ranged in experience from beginner to elite, with most having university level athletic experience or higher, and represented a variety of sports. Noetel and colleagues suggest based on their survey of the mindfulness literature in sport that, although studies conducted to date have revealed positive outcomes of mindfulness-based interventions, the evidence is presently quite low in quality. Despite finding large effect sizes for mindfulness and acceptance-based interventions in facilitating performance, flow, and presentmoment awareness, the quality of the findings was impacted by bias risk across studies and effect size imprecision. Preliminary support was offered for the use of mindfulness-based 
interventions in the prevention of injuries, reduction of burnout, and increase of confidence among athletes. Observational or correlational studies revealed that athletes tend to vary in their degree of mindfulness, and that a higher proclivity toward mindfulness may be linked to lower stress and ratings of perceived exertion, and higher self-efficacy, self-determined motivation, and mental toughness.

Despite consolidating a larger collection of studies from which to pool evidence than have past reviews (e.g., Sappington \& Longshore, 2015), stronger conclusions regarding the effectiveness of mindfulness and acceptance-based approaches for enhancing athletic performance were unable to be drawn (Noetel et al., 2017). Thus, there is a continued need for further studies with rigorous methodological and study design features at the fore. The authors also note that, although mindfulness-based approaches may offer benefits over no treatment, future researchers can contribute to the extant mindfulness literature by comparing such approaches with other well-established interventions (e.g., goal setting, mental practice, self-talk) that aim to manage attention or modulate the content of internal experiences. This would allow for the side-by-side comparison of mindfulness and acceptance-based approaches to other PST approaches to performance enhancement in sport. Limitations to pooling data across various studies that examined different outcomes (e.g., mindfulness, flow, performance) and different interventions (mindfulness and acceptance) precluded meta-analytic procedures. Limitations notwithstanding, the authors synthesized the findings from a larger number of studies than in recent years to offer recommendations for future research directions - primarily related to the design of high quality studies that allow causal conclusions to be drawn regarding the effectiveness of mindfulness and acceptance-based approaches in sport. 
In another recent study, Bühlmayer, Birrer, Röthlin, Faude, and Donath (2017) conducted a meta-analytic review to assess the effects of mindfulness on performance-relevant parameters and on performance outcomes in sport. Following exclusion criteria, nine mindfulness-based intervention trials were included in the study. The 290 athletes across the included trials were over 15 years of age, and participated in a range of different sports including cycling, dart and hammer throwing, hockey, judo, rugby, running and track and field, shooting, tennis, and volleyball. The researchers found primarily large effects of mindfulness on both physiological (e.g., cortisol level) and psychological (e.g., anxiety) parameters relevant to performance, and on indices of performance outcomes. The findings pertaining to outcomes of performance were derived solely from precision sports (i.e., dart throwing, shooting), suggesting that further research is needed to clarify the performance facilitating effects of mindfulness in a broader range of sport outcomes.

At the single study level, preliminary support for mindfulness-based interventions in sport has been offered through randomized trials. For example, Moghadam, Sayadi, Samimifar, and Moharer (2013) showed that adult male Iranian Premier League badminton athletes $(n=20)$ who underwent a mindfulness education program had significantly higher post-intervention performance $(M=47.0)$, as measured objectively by scores in five matches, compared to control group athletes $(n=20 ; M=43.7)$. Post-intervention competitive anxiety levels were also significantly lower for athletes in the experimental group $(M=27.6)$ than in the control group $(M$ $=33.3$ ). The authors reported that the mindfulness training intervention accounted for $37 \%$ of the variance in sport performance. Despite these encouraging findings, an explanation of the nature and duration of the mindfulness intervention was lacking, as was information pertaining to a potential alternative task among control group participants, both of which present limitations to 
the practical utility of the results. For replication and extension efforts to be feasible, researchers are encouraged to present intervention details with adequate depth and clarity.

John, Verma, and Khanna (2011) examined the effect of mindfulness meditation on elite male shooters' $(N=96)$ performance and pre-competition anxiety. The intervention was composed of mindfulness meditation exercises, including focused breathing, body scanning, and restorative yoga poses. The intervention group $(n=48)$ engaged in these exercises in 20 minute sessions, six days per week, for four weeks. Pre-competition anxiety was measured via salivary cortisol levels, a physiological marker of anxiety/stress, while performance was measured based on shooting accuracy scores. Elite shooters in the experimental group experienced significant increases in shooting performance and significant decreases in pre-competition anxiety at posttest and follow-up measures, compared to the control group. Due to the absence of an alternative or distraction task for the control group, one is unable to determine whether the performance effects might have occurred due to the increased attention paid toward athletes in the experimental group or to some other confounding variable. Despite this limitation, the use of an objective measure of sport performance at an elite level of athletics has implications for future researchers looking to strengthen their methodological designs through the use of similar performance measurements in sports where individual scores are made available.

Aherne, Moran, and Lonsdale (2011) conducted a mindfulness training intervention to explore flow experiences and cognitive-affective mindfulness among 13 male and female Irish collegiate athletes in a variety of sports (i.e., field hockey, hammer throw, hurdling, rugby, running, and tennis), and ranging in age from 19 to 25 . Athletes in the intervention group $(n=6)$ received information about the history and theoretical underpinnings of mindfulness, and participated in a guided, self-directed mindfulness training program (educational/instructional 
video recordings) across six weeks. The researchers found that athletes in the experimental group $(M=151.00)$ had significantly higher global flow scores post-intervention than did athletes in the control group $(M=131.57)$. In addition, athletes who participated in the mindfulness intervention showed significant increases in global flow experiences from baseline to follow-up measurements, whereas such differences were not observed among athletes in the control group. Cognitive-affective measures of mindfulness were also significantly higher for athletes who underwent the mindfulness intervention compared to athletes in the control group. Although the authors thoroughly explained the nature of the protocol employed with the mindfulness intervention group, the relatively small sample of athletes raises concerns regarding power to detect true differences in the study. Furthermore, despite the prevailing notion that flow facilitates optimal performance in sport, the lack of direct performance measures in the current study leaves the mindfulness-performance relationship to speculation. Taken together, Moghadam et al. (2013), John et al. (2011), and Aherne et al. (2011) suggest that mindfulnessbased practices may indeed facilitate sport performance and other key psychological aspects of sport. Still further research with greater methodological rigor (e.g., larger sample sizes, randomization, longer treatment periods, and alternative treatments/tasks for control group participants) is needed to provide greater support for the performance enhancing effects of mindfulness in sport.

Beyond this collection of randomized controlled trials, features of several recent studies in the mindfulness literature were deemed particularly relevant to the present review given their focus on objective performance measures, flow, and/or aspects of perfectionism. For instance, a series of studies was conducted by Bernier et al. (2009) to (1) explore associations between mindfulness, acceptance, and flow experiences in sport, and (2) assess the effectiveness of a 
mindfulness and acceptance based program on competitive sport performance. The first study was qualitative and exploratory in nature, and aimed to draw out experiences of flow among 10 elite swimmers using semi-structured interviews. The researchers found that elements of optimal sport experiences described among the swimmers were closely related to dimensions of flow (Jackson \& Csikszentmihalyi, 1999), with autotelic experience, total concentration, challengeskill balance, sense of total control, and unambiguous feedback arising most frequently within the interviews. Additionally, a heightened state of body awareness emerged in the swimmers' optimal performance accounts, often through indications of acceptance rather than avoidance of bodily sensations. The findings of study one support the notion that characteristics of mindfulness and acceptance appeared to be linked to flow and athletes' optimal sport experiences.

In the second study, Bernier et al. (2009) aimed to test the effectiveness of a mindfulness and acceptance based approach to PST compared to a traditional change-based PST program for the enhancement of sport performance and development of mental skills among young elite golfers. The mindfulness and acceptance based intervention drew from elements of MBCT and ACT, and was delivered to the athletes following the development of traditional psychological skills (i.e., goal setting, activation, concentration, relaxation, and imagery). The researchers discovered that all athletes in the intervention group $(n=7)$ enhanced their national ranking, compared to just two athletes in the traditional PST program control group $(n=6)$. Furthermore, athletes involved in the mindfulness and acceptance based intervention showed significantly greater increases in their activation skills compared to athletes in the control group, and expressed via qualitative interviews the development of nonjudgmental awareness, task-relevant focus of attention, and behavioral flexibility. Taken together, the researchers revealed that 
mindfulness and acceptance approaches are closely linked to flow and optimal performance in sport, and can be used for athletic performance enhancement. Further, the authors call into question the effectiveness of purely traditional PST approaches to sport performance enhancement. However, it must also be noted that the study had a small sample size, and was likely underpowered leading to a possible Type II error in the research. Studies involving larger sample sizes are thus needed to provide greater confidence in the extant mindfulness findings.

As briefly noted above, Kee and Wang (2008) conducted a study to explore relationships among mindfulness, flow, and the adoption of mental skills among university student athletes ( $N$ =182). A cluster analytic approach was used to create profiles of athletes who possess similar mindfulness characteristics. The researchers revealed a unique four cluster solution based on athletes' mindfulness characteristics, as assessed using the Langer Mindfulness/Mindlessness Scale (MMS; Bodner \& Langer, 2001), including one high mindfulness profile, and one low mindfulness profile, with two more moderate profiles on the mindfulness constructs assessed. Athletes in each of these profiles were then compared based on levels of flow assessed using the Dispositional Flow Scale (DFS-2; Jackson \& Eklund, 2004), and on mental skill adoption as assessed using the Test of Performance Strategies (TOPS; Thomas, Murphy, \& Hardy, 1999). Findings revealed that athletes with profiles high in mindfulness reported significantly higher flow dispositions (i.e., loss of self-consciousness, challenge-skill balance, sense of control, concentration, and clear goals) and higher adoption of mental skills (i.e., goal setting, attentional and emotional control, and self-talk) compared to athletes with profiles low in mindfulness. The researchers suggest that mindful athletes may have a greater propensity to experience flow and to adopt mental skills in sport. 
Although some of the recent empirical literature discussed herein has examined links between mindfulness and sport performance, relatively little has investigated this relationship relative to objective measures of performance. Gooding and Gardner (2009) assessed the association between mindfulness and basketball free throw shooting percentage, along with preshot routine duration and anxiety, in a sample of NCAA Division I men's basketball players $(N=$ 17) ranging in age from 19 to 24 years. Of particular relevance to the current review, the researchers hypothesized that mindfulness would arise as a significant predictor free throw shooting percentage in regular season games. Prior to the start of the basketball season, athletes were administered the Mindful Attention Awareness Scale (MAAS; Brown \& Ryan, 2003), and the Sport Competition Anxiety Test (SCAT; Martens, Vealey, \& Burton, 1990). Copies of the regular season game tapes were provided to the primary researcher at the end of the season to analyze the free throw attempts taken by each athlete, as well as the duration of their pre-shot routines. Linear regression analysis revealed that basketball players' levels of mindfulness did indeed significantly predict free throw percentage in games across the competitive season, with a one standard deviation increase in mindfulness resulting in a 5.75 percent increase in free throw shot percentage. Furthermore, mindfulness significantly predicted the difference between individuals' practice and game free throw performance. When competitive experience (i.e., year in school) was controlled for through hierarchical regression analysis, however, mindfulness no longer arose as a significant predictor of free throw performance.

Based on these findings, Gooding and Gardner (2009) suggested that levels of mindfulness among collegiate athletes - a sample who did not receive any previous training in mindfulness-based approaches - significantly predicted objectively measured competitive performance. Given the positive relationship between mindfulness and real-world sport 
performance among collegiate basketball players, future research might aim to extend such findings into other sport contexts where objective measures of performance can feasibly be obtained. The results must be interpreted with noteworthy caveats, however, given that (1) mindfulness failed to arise as a significant predictor of performance over and above competitive experience (indicating some degree of shared variance between competitive experience and mindfulness), and (2) such a positive relationship between mindfulness and performance does not necessarily indicate that mindfulness caused performance improvements. On a final note, scholars have posited the importance of context specificity in measurement. Because the present study used a non-sport specific measure, researchers might consider incorporating a sportspecific measure of mindfulness into future studies to support and extend our understanding of mindfulness - performance relationships in the context of sport.

Another series of studies in the mindfulness and sport literature have focused on a newly developed sport-specific mindfulness-based approach that integrates teachings from both MBSR and MBCT. Kaufman et al. (2009) evaluated how their MSPE approach to mental training might influence sport performance, flow experience, and other psychological factors commonly associated with performance among male and female recreational level archers $(n=11)$ and golfers $(n=21)$. Athletes engaged in a four-week MSPE program, completed measures of state mindfulness (Toronto Mindfulness Scale, TMS, Lau et al., 2006) and state flow (Flow State Scale-2, FSS-2, Jackson \& Eklund, 2002) during the intervention, and completed a series of trait measures pre- and post-intervention (Multidimensional Perfectionism Scale, MPS, Frost, Marten, Lahart, \& Rosenblate, 1990; Thought Occurrence Questionnaire for Sport, TOQS, Hatzigeorgiadis \& Biddle, 2000; Kentucky Inventory of Mindfulness Skills, KIMS, Baer, Smith, \& Allen, 2004; Dispositional Flow Scale-2, DFS-2, Jackson \& Eklund, 2002). Athlete 
participants also kept self-report mindfulness and sport performance logs, in addition to completing an initial background questionnaire and a final exit survey.

The researchers found that sport performance satisfaction among athletes was significantly higher post-mindfulness program than at pre-program, $t=3.24, p<.01$ (Kaufman et al., 2009). Overall levels of state flow reported among the athletes significantly changed over the course of the MSPE training. Furthermore, sport anxiety $(r=-.72)$, perfectionism $(r=-.75)$, and thought disruption $(r=-.73)$ had significant negative relationships with overall levels of flow; whereas, mindfulness $(r=.79)$ and confidence $(r=.72)$ had significant positive relationships with overall flow levels (Kaufman et al.). Thus, preliminary support was obtained for associations among mindfulness and a variety of psychological aspects of sport thought to be linked to optimal performance experiences. Although mindfulness was significantly associated with flow, the relationship between mindfulness and sport performance was not significant. Lack of power resulting from a small sample size, and the relatively short mindfulness intervention duration may account for the lack of performance findings.

In another sport context, De Petrillo, Kaufman, Glass, and Arnkoff (2009) sought to examine how a sport-specific mindfulness-based intervention might influence the performance of long-distance runners, as well as anxiety and perfectionism. the researchers recruited recreational long-distance runners $(N=25)$ who competed in races with regularity. Athletes in both the MPSE intervention $(n=13)$ and waitlist control groups were asked to document their running performance by keeping a log across the four-week duration of the study, and athletes completing the intervention were asked to keep a mindfulness practice log. Extensive self-report measures were administered to athletes pre- and post-intervention, and included a background 
questionnaire, the Sport Anxiety Scale (SAS; Smith, Smoll, \& Schutz, 1990), TOQS, MPS, KIMS, TMS, and the Credibility and Expectations Measure (CEM; Kaufman et al., 2009). The researchers found that long-distance runners who participated in the workshop reported significantly lower organizational demands $(M=22.17)$, a subscale of the MPS perfectionism measure, than did runners in the control group $(M=24.90)$. The authors indicated this result might be due to mindfulness impacting athletes' ability to accept imperfection and thus lowering athletes' overemphasis on rigid organization, neatness, and precision. However, the significant link between mindfulness practice and organizational demands might very well have occurred due to chance, given that this was the only significant difference between groups. Apart from this finding, long-distance runners in the workshop group did not significantly differ from control group runners on most other measures. As in the previous study, relatively short mindfulness program duration, small sample size, and recreational sport level were offered as possible explanations for the lack of significant differences between groups of participants.

When intervention and control participants were combined, however, significant changes from pre- to post-intervention were found. Both dispositional mindfulness and state mindfulness showed significant increases, and sport anxiety worries and aspects of perfectionism (i.e., parental criticism and personal standards) showed significant decreases from pre- to postworkshop. Although sport performance was not significantly enhanced through participation in the mindfulness workshops, this may be attributable to the newness of the skills and the short duration in which to integrate the skills into their long-distance running performance. Despite the lack of performance improvement during the study, a vast majority (81\%) of the athletes reported that continuing to practice mindfulness would both help them improve their performance and help them cope with stress. Though the authors feel the data offer support for 
MSPE as a program for increasing mindfulness and for decreasing sport anxiety worries and dimensions of perfectionism among athletes, there are substantial limitations in the design and outcomes that are concerning, particularly related to the small sample size, low degree of power, and lack of performance improvements which ultimately is a key aim of the MSPE approach.

Along the same line of research, Thompson et al. (2011) aimed to examine the longerterm impact of mindfulness-based programming for sport performance and other psychological aspects of sport. Due to the relatively brief four-week MSPE program used by Kaufman et al. (2009) and De Petrillo et al. (2009), it is not surprising that significant changes in sport performance were elusive. Thompson et al. (2011) thus deemed a follow-up examination of the sport-specific mindfulness-based intervention important for understanding its effect on sport performance enhancement. Athlete participants were archers $(n=4)$, golfers $(n=8)$, and longdistance runners $(n=13)$ who participated in the original MSPE workshops offered by Kaufman et al. and De Petrillo et al. Participants $(N=25)$ were both men $(n=14)$ and women $(n=11)$, ranging in age from 18 to 72 years $\left(M_{\text {age }}=48.28\right.$ years). Sport-specific, follow up, and workshop credibility questionnaires, as well as a comparable series of trait measures employed in the original study protocols, were returned by consenting athletes one year post-intervention.

The researchers revealed significant performance improvement in the mile times of longdistance runners, from both pre- and post-test to follow-up (Thompson et al., 2011). Although the golfers' 18-hold round scores did not change significantly from pretest to follow up, the golfers' scores significantly decreased from posttest to follow up. Changes in the archers' performance were not assessed due to the small number of participants who completed the follow-up assessments. The authors recommended that future researchers consider using average measures of athletic performance rather than measures of athletes' best performances to foster 
greater validity. Limitations might also exist regarding the degree to which one can attribute performance improvements post-workshop to the intervention itself, and not purely to improvements in sport-specific skill and technique. Small sample size and athletes self-selecting to participate in the current follow-up study were noted as limiting the power and generalizability of the findings, respectively. Such limitations notwithstanding, the authors offered that the significant associations between athletic performance improvements and increased mindfulness found in the study lend support for the notion that optimal performance in sport may be attained through acceptance and present moment awareness, rather than through the conscious exertion of control over internal experience.

Reductions in aspects of perfectionism, including overall trait perfectionism, concern over mistakes, and doubting of actions, were also found among athletes at follow-up (Thompson et al., 2011). Mindfulness may therefore contribute to reductions in dysfunctional dimensions of perfectionism. Given that performance improvements were found to be negatively associated with overall trait perfectionism $(r=.74)$, concern over mistakes $(r=.69)$, and doubts about actions $(r=.75)$, the researchers offered additional support to the notion that aspects of perfectionism were found to be linked to suboptimal performance in sport. It is important to note that negative relationships are reflected in the positive correlations reported because performance improvement is measured through decreased mile time. Due to the correlational nature of the study, the authors suggested interpreting the link between perfectionism and performance with care. Taken together, the findings of Thompson et al. provide additional credence to the understanding that mindfulness may have performance benefits, and that certain dimensions of perfectionism may negatively influence athletic performance improvements.

\section{Perfectionism in Sport and Performance Psychology}


Perfectionism has been defined as "a personality disposition characterized by striving for flawlessness and setting exceedingly high standards for performance, accompanied by tendencies for overly critical evaluations" (Stoeber, 2012, p. 294). Two primary frameworks of perfectionism have been documented in the literature, both of which support the multidimensional nature of the phenomenon. Frost et al. (1990) offered a six-facet conceptualization of perfectionism, consisting of concern over mistakes, doubts about actions, personal standards, organization, and both parental expectations and criticism. Another framework posited by Hewitt and Flett (1991) organized the construct of perfectionism into three primary facets including self- and other-oriented perfectionism, and socially prescribed perfectionism.

Whether perfectionism exists as a three facet, six facet, or some other facet structure phenomenon, researchers have generally accepted perfectionism as a multidimensional construct. A more controversial point of discussion, however, is whether certain dimensions of perfectionism may be adaptive and others maladaptive (Flett \& Hewitt, 2005). In their discussion on conceptions of perfectionism, Stoeber and Otto (2006) synthesized the perfectionism frameworks outlined above through two higher-order dimensions - perfectionistic strivings and perfectionistic concerns. Perfectionistic strivings relate to one's proclivity to establish high standards and strive for excellence (i.e., organization, personal standards, self- and other-oriented perfectionism). In contrast, perfectionistic concerns relate to one's tendency to be self-critical, and fearful of making mistakes or being evaluated negatively (i.e., concern over mistakes, doubts about actions, parental expectations and criticism, and socially prescribed perfectionism).

Stoeber and Otto (2006) asserted that these two higher-order dimensions of perfectionism may help distinguish among three types of perfectionists: non-perfectionists, who have low 
levels of perfectionistic strivings; healthy or adaptive perfectionists, who tend to display low perfectionistic concerns and high perfectionistic strivings; and unhealthy or maladaptive perfectionists, who have high levels of both perfectionistic concerns and strivings. In addition to this tripartite model of perfectionism, a 2 x 2 model of perfectionism has also been suggested. The model further differentiates what was formerly considered non-perfectionists into two profiles: individuals who are low in perfectionistic strivings and high in perfectionistic concerns, and individuals who are low in both strivings and concerns (Gaudreau \& Thompson, 2010).

Despite the different models of perfectionism that have arisen in the literature, perfectionism dimensions are often correlated, meaning that individuals with high levels of strivings may also have high levels of concerns. When researchers controlled for shared variance between strivings and concerns, for instance, unique relationships became more pronouncedevidence for perfectionistic strivings being adaptive has increased (Stoeber \& Otto, 2006), as has evidence for perfectionistic concerns being maladaptive (Stoeber, 2014). Each dimension of perfectionism therefore tends to have a contrasting association with performance outcomes once shared variance has been taken into consideration. More specifically, and a point of much discourse among scholars in recent years, perfectionistic strivings have often been positively associated with performance; whereas, perfectionistic concerns have often been negatively associated with performance (e.g., Stoeber, 2012). Nuances have arisen, however, regarding perfectionism - performance relationships among athletes.

Much agreement has been reached regarding the maladaptive nature of perfectionistic concerns in sport; however, scholars studying the adaptive vs. maladaptive nature of perfectionistic strivings have arrived at contrasting conclusions. Flett and Hewitt (2005) asserted in their article on the 'perils' of perfectionism that possessing characteristics of perfectionism is 
predominantly maladaptive and does not tend to facilitate desirable outcomes in sport. The authors put forth the notion of a perfectionism paradox - that although some sports require athletes to strive toward perfect performance, preoccupation with attaining perfection often undermines sport performance outcomes. Gotwals, Stoeber, Dunn, and Stoll (2012) sought to better understand whether perfectionistic strivings among athletes are adaptive or maladaptive by surveying much of the extant literature to examine correlations across over 30 studies on perfectionism among athletes. Accounting for shared variance with perfectionistic concerns, a majority of evidence linked perfectionistic strivings with adaptive aspects of sport, while a minority linked perfectionistic strivings with maladaptive aspects. Still further evidence showed nonsignificant correlations between perfectionistic strivings and both adaptive and maladaptive aspects of sport. Collectively, once shared variance with perfectionistic concerns was controlled, correlational trends suggested that perfectionistic strivings are rarely maladaptive, sometimes neutral, and mostly adaptive among athletes.

In a more recent meta-analysis of multidimensional perfectionism in sport, Hill, Mallinson-Howard, and Jowett (2018) sought to provide an updated review of the literature and to further clarify relationships between perfectionistic strivings and perfectionistic concerns among athletes. Based on the 52 studies included in the meta-analytic review, the researchers found that perfectionistic concerns were clearly maladaptive, while perfectionistic strivings tended to be more complex. Perfectionistic strivings were related to a blend of both maladaptive and adaptive psychological outcomes (e.g., self-confidence, cognitive anxiety) and better athletic performance; whereas, perfectionistic concerns were predominantly related to maladaptive psychological outcomes (e.g., cognitive and somatic anxiety) and were not related to performance. 
When shared variance between strivings and concerns was accounted for, residual perfectionistic strivings and residual perfectionistic concerns showed more pronounced profiles (Hill et al., 2018). For example, residual perfectionistic strivings displayed a more adaptive profile of relationships to psychological outcomes than did perfectionistic strivings. In contrast, residual perfectionistic concerns showed a more maladaptive profile of relationships to psychological outcomes than did perfectionistic concerns. The researchers concluded that while perfectionistic strivings may benefit athletic performance in some circumstances, perfectionism both strivings and concerns - may be a vulnerability factor for athletes. These findings underscore the importance of accounting for relationships between dimensions of perfectionism when aiming to understand their unique effects on psychological and performance outcomes.

Researchers have employed person-centered approaches to better understand individual profiles of perfectionism in sport, and whether distinct groups of individuals might arise along various dimensions of perfectionism. For example, Martinent and Ferrand (2006) employed a cluster analytic approach among a sample of competitive athletes $(N=166)$ and found three unique profiles of perfectionists (i.e., adaptive, maladaptive, and non-perfectionists) based on the Sport Multidimensional Perfectionism Scale (Sport-MPS; Dunn et al., 2002, 2006) and the Multidimentional Perfectionism Scale (MPS; Hewitt \& Flett, 1991). More recently, Sapieja, Dunn, \& Holt (2011) supported the three-cluster conceptualization of perfectionism revealed by Martinent and Ferrand using the more recent Sport Multidimensional Perfectionism Scale-2 (Sport-MPS-2; Gotwals \& Dunn, 2009) among male youth soccer players $(N=194)$. Upon replicating the methods employed by Sapieja and colleagues, Gotwals (2011) further distinguished the presence of a second maladaptive perfectionist profile among varsity athletes $(N=117)$. The two maladaptive perfectionist profiles were differentiated based on levels of 
parent expectations/criticisms and doubts about training/competition. In another study using a cluster analytic approach, this time among male youth ice hockey players $(N=229)$, Vallance, Dunn, and Causgrove Dunn (2006) supported a three-cluster solution (i.e., low, moderate, and high) based on the four primary dimensions of the Sport-MPS. In sum, these studies confirm the presence of unique perfectionism profiles among athletes. The samples accessed were either relatively small or composed solely of male youth athletes, however, and thus generalizing the findings to other specific sport populations (e.g., female adult athletes) must be cautioned.

In an effort to extend person-centered perfectionism research to a diverse sample of high level youth and adult athlete participants, Gucciardi, Mahoney, Jalleh, Donovan, and Parkes (2012) explored profiles of perfectionism and motivational orientations among elite male ( $n=$ $179)$ and female $(n=244)$ athletes ranging in age from 14 to $66(\mathrm{M}=25.64, \mathrm{SD}=8.57)$, and representing both team (e.g., baseball, rugby, hockey, rowing) and individual (e.g., athletics, cycling, gymnastics, triathlon) sports. Based on the acknowledged importance of using domainspecific measures, particularly in sport, individuals were clustered based on responses to the Sport-MPS. The measure assesses perfectionism in sport based on perceived parental and coach pressures, personal standards, and concern over mistakes. The two-step cluster analysis consisted of a hierarchical Ward's method to determine an initial number of perfectionism clusters represented, followed by a nonhierarchical $k$-means cluster analysis to specify the cluster solution identified using the hierarchical approach. A MANOVA was then used to explore differences across the clusters identified on the motivational orientation dependent variables assessed (i.e., mastery approach/avoidance and performance approach/avoidance goals, fear of failure, external regulation, and intrinsic motivation). 
Consistent with existing literature, Gucciardi et al. (2012) found support for the profiles of adaptive/healthy perfectionists, maladaptive/unhealthy perfectionists, and non-perfectionists, representing a tripartite conceptualization of perfectionism. The adaptive and maladaptive perfectionist profiles both showed high levels of personal standards; however, individuals in the maladaptive perfectionist profile also showed high levels of perceived parental and coach pressures and concern over mistakes. Thus, the researchers concluded that high personal standards for performance is likely not a maladaptive facet of perfectionism unless combined with critical self-evaluations or critical evaluations from important others. Additionally, the researchers revealed noteworthy differences in motivational orientations among the perfectionism profiles. Specifically, the adaptive perfectionists reported significantly higher levels of mastery approach goals $(z=.29)$, and lower levels of fear of failure $(z=-.20)$ and performance avoidance $(z=-.12)$, mastery avoidance $(z=-.07)$, and performance approach $(z=-$ .01 ) goals than maladaptive perfectionists (respectively, $z=-.02, .74, .48, .49, .58$ ). Furthermore, non-perfectionists reported significantly lower levels of intrinsic motivation $(\mathrm{z}=-.35)$, mastery approach goals $(\mathrm{z}=-.37)$, and performance approach goals $(\mathrm{z}=-.51)$ than adaptive perfectionists (respectively, $\mathrm{z}=.24, .29,-.01$ ), as well as lower levels of the seven motivational orientations measured than maladaptive perfectionists.

The Gucciardi et al. (2012) findings highlight the ability to distinguish differences among perfectionism profiles on various dependent variables of interest; however, the application of these findings is significantly limited without specific behavioral measures of performance. Thus, although research examining profiles of perfectionism among athletes deepens our understanding of the multidimensional nature of the construct, our understanding of the link between those perfectionistic profiles and performance outcomes ultimately depends upon 
inclusion of behavioral performance measures in future research designs. Despite the methodological strength of recruiting a relatively large sample of elite athletes representing a broad range of sports, the researchers also highlighted that future researchers might consider measuring perfectionism using the more recent Sport-MPS-2 to improve upon the methodology employed in the present study.

In a variable-centered approach to the topic, Stoeber, Uphill, and Hotham (2009) sought to systematically examine relationships between dimensions of perfectionism and competitive sport performance among triathletes. Specifically, the researchers conducted a series of two studies to investigate (1) how perfectionism might affect triathlon performance, and (2) the role that athletes' goals might play in the perfectionism - performance relationship. A sample of 112 male and female athletes completed study 1, with participants ranging in age from 21 to 58 years $(M=36.5, S D=7.6)$ being recruited from a Half-Ironman distance triathlon event. Perfectionism was measured using the personal standards and concern over mistakes subscales of the SportMPS tailored to individual sport athletes. Researchers used the Achievement Goals Questionnaire for Sport (AGQ-S; Conroy, Elliot, \& Hofer, 2003) to measure four types of goals combining mastery-performance with approach-avoidance dimensions. Triathlon performance data was obtained from official race records, and athletes self-reported best triathlon times from the season, in addition to season best times in each of the swimming, cycling, and running distances. Of particular note, results of the hierarchical regression and mediation analyses indicated the following: (1) athletes higher in personal standards perfectionism had faster race times than those lower in personal standards perfectionism, controlling for differences in performance level; (2) athletes higher in performance-approach goals had faster race times than those lower in this motivation style; (3) athletes higher in performance-avoidance goals had 
slower race times than those lower in this motivation style; and (4) the relationship between personal standards perfectionism and race performance was mediated by the athletes' achievement goals.

Participants in study 2 were 321 male and female athletes ranging in age from 19 to 67 years $(M=37.2, S D=7.9)$ who were recruited from Olympic distance triathlons (Stoeber et al., 2009). Measures used to assess perfectionism, performance, and achievement goals were the same as described in study 1 . Results of the hierarchical regression and mediation analyses with this larger sample of athletes at a longer race distance replicated the primary findings of the previous study. Taken together, the studies support personal standards perfectionism as a positive predictor of competitive sport performance. These findings suggest that aspects of perfectionism may facilitate performance in real-world contexts. Furthermore, athletes' achievement goals and motivations appear to impact the link between perfectionism and performance in sport.

In sum, personal standards perfectionism may influence athletes' healthy strivings for excellence in sport, and this contribution may be influenced by other factors, including athletes' performance goals. Although achievement goal orientations are beyond the scope of the present discussion, Stoeber et al. (2009) support the notion that relationships among psychological constructs, including perfectionism, are often complex. The present study looked only at triathlon athletes, and we must therefore use caution when attempting to generalize the perfectionism - competitive performance relationship to other sport populations. However, in light of recent findings that higher levels of competitive sport tend to be associated with higher levels of perfectionistic strivings (Rasquinha, Dunn, \& Causgrove Dunn, 2014), the results of Stoeber and colleagues' studies linking perfectionistic strivings to favorable performance outcomes may have implications for athletes in high levels of competitive athletics. Given the 
relative agreement that maladaptive aspects of perfectionism tend to be negatively linked to sport performance outcomes and psychological aspects of sport, further research could help to elucidate the picture of perfectionism and real-world competitive performance in sport.

In light of the literature presented, it appears as though some agreement has been reached regarding perfectionism and its links to characteristics of sport: (1) perfectionism is a multidimensional construct, or perhaps a multidimensional construct with a smaller number of higher-order general factors; (2) dimensions of perfectionistic concerns have often been negatively linked to psychological aspects and behavioral outcomes in sport; (3) perfectionistic strivings have had mixed associations with psychological aspects and behavioral outcomes in sport, yet seem to be primarily adaptive; (4) unique profiles of perfectionism have arisen among high level athletes, and these have been used to predict sport-related variables. Consideration of key variables presented throughout the current review (e.g., mindfulness, flow, perfectionism, and performance) might illuminate potential pathways of influence, and identify areas for future research.

In their chapter on mindfulness and flow in sport, Baltzell and Diehl (2017) outline a conceptual model to visually represent the mindfulness-flow-performance links in sport. In the model, mindfulness is depicted as a pathway to move from a non-present mind state to a more present-mind state. In that present-mind state, one opens the door to experiencing flow and adaptive performance. Moderators of adaptive performance include wise discernment, psychological skill training, and facilitators of flow. Within the non-present mind state, one may have experiences of distraction, performance anxiety, harsh self-criticism, fear, and anger, among others, and this non-present mind state is shown to be linked to poor performance and/or avoidance. 
In the Mindfulness-Flow-Performance Model in Sport described (Baltzell \& Diehl, 2017), the link between mindfulness, flow, and adaptive performance outcomes are made quite clear. Mindfulness and flow have been positively associated both with one another, and with performance in sport. Where then might perfectionism fit into the model? Included in the nonpresent mind state is the experience of harsh self-criticism, often closely linked to perfectionistic concerns. Perhaps then, maladaptive characteristics of perfectionism might be linked to a nonpresent mind state, and mindfulness might bring individuals with perfectionistic concerns closer to states facilitative of performance in sport (i.e., present mind state, flow). If we consider perfectionistic strivings as adaptive, as much of the recent literature has suggested, then perhaps the adaptive striving characteristics of perfectionism might be included as a moderator of adaptive performance. In light of the contrasting influences found between perfectionistic concerns and perfectionistic strivings, linking these different dimensions of perfectionism to different elements of our model seems justified. Furthermore, aspects of perfectionism have been negatively associated with flow, and mindfulness has been positively associated with flow. Therefore, perhaps mindfulness serves as a key link in the model, facilitating adaptive performance both via reductions in maladaptive characteristics of perfectionism (and a move from a non-present to present mind state), and via increases in flow experiences. Associations among mindfulness, flow, perfectionism, and performance have been discussed throughout the present review, and multiple pathways of influence supported in the empirical literature may in turn be represented through the theoretical model described.

\section{Measurement}

The development of psychometrically sound measures in quantitative research is essential to the integrity of empirical literature. In order to have confidence in published research, the 
instruments underlying our reported findings must be both valid and reliable. The current section will outline measurement of mindfulness, perfectionism, and performance, both broadly and specific to sport.

\section{Mindfulness}

In tandem with the expansion of mindfulness literature in recent years, researchers have developed a variety of instruments to measure mindfulness, both general and sport-specific. Sauer et al. (2013) and Bergomi, Tschacher, and Kupper (2013) reviewed a series of self-report measures of mindfulness. Between the two reports, the authors discussed 11 general mindfulness assessments as outlined in Table 1. Although a comprehensive review of the available mindfulness measures is beyond the scope of the current discussion, one may see plainly based on the extensive list of self-report measures outlined above that mindfulness and its assessment have received great interest in recent years. When deciding upon which of the many mindfulness measures to use, one might be wise to consider (1) the aspects/dimensions of mindfulness assessed, (2) the theoretical framework from which the measure was developed (e.g., Eastern or Western conceptualization), (3) whether the instrument measures state- or trait- mindfulness, (4) and the psychometric properties of the developed assessments. Keeping these considerations in mind will allow future researchers to sort among the many scales to determine which measure of mindfulness might have greatest utility in a given study.

Given that the MAAS (Brown \& Ryan, 2003) was used to help establish construct validity when developing the recent sport-specific measures of mindfulness discussed below, the MAAS warrants further mention. The MAAS is a 15-item, single-factor instrument assessing the typical frequency of mindfulness characteristics among individuals over time. As such, the instrument may be regarded as measuring trait-mindfulness. Items are rated on 6-point Likert 
scales, ranging from 1 (almost always) to 6 (almost never). Participants are asked to indicate how frequently they have the experience described in each statement. Items span physical, interpersonal, cognitive, emotional, and general domains, with the focus across items placed on how frequently one experiences attention and awareness in the present moment. Factor loadings ranged from .27 to .78 for the 15 items, and items with low factor loadings were selected to be retained because they contributed substantively to the measure. Fit indices were considered satisfactory for both the university student sample, $X^{2}(90)=189.57, \mathrm{GFI}=.92, \mathrm{CFI}=.91$, IFI $=$ $.91, \mathrm{PCFI}=.78, \mathrm{RMSEA}=.06$, and the general adult sample, $\mathrm{X}^{2}(90)=179.14, \mathrm{GFI}=.91, \mathrm{CFI}=$ $.92, \mathrm{IFI}=.92, \mathrm{PCFI}=.79, \mathrm{RMSEA}=.07$. Internal consistency (alpha) for the student and adult samples were .82 and .87 , respectively. Test-retest reliability was examined over a 4-week period, and the temporal stability of the measure was established. Convergent and discriminant validity of the MAAS were supported through significant associations with conceptually similar (i.e., attention to emotions, mood repair, emotional intelligence, openness to experience, clarity of emotional states, internal state awareness, and elements of mindfulness) and dissimilar (e.g., public self-consciousness, social anxiety, and rumination) constructs. Comparative validity revealed that both indirect- and direct-worded versions of the MAAS essentially measured the same construct, and thus the initial indirect-worded items of the scale were retained.

The context of sport is quite distinct from the context of everyday life, particularly in terms of the demands placed on attention and awareness in athletic performance. As such, measures of mindfulness specific to the context of sport are needed to accurately reflect mindfulness experiences among athletes. Two recent mindfulness measures have been developed for athlete participants. The Mindfulness Inventory for Sport (MIS; Thienot et al., 2014) is a 15item, 3-factor measure that also assesses mindfulness in the context of sport. The subscales 
include non-judgmental attitude, awareness, and refocusing. Items are measured by 6-point Likert scales, ranging from 1 (not at all) to 6 (very much), and are stemmed by the following phrase: "The statements below describe a number of things that athletes may experience just before or during their sport performance. Please circle the number that best indicates how much each statement is generally reflective of your recent experience. There are no right or wrong answers." Considering the language included in the instrument stem (i.e., generally reflective), the measure may be regarded as trait-like in nature. The 15-item, 3-factor structure of the model provided an adequate fit to the data, $X^{2}(87)=166.98, \mathrm{p}<.001, \mathrm{CFI}=.93$, IFI $=.93$, TLI $=.91$, $\mathrm{SRMR}=.06, \mathrm{RMSEA}=.05$. Construct validity was supported through significant correlations with conceptually similar and dissimilar constructs (i.e., MAAS, flow, rumination, worry, concentration disruption, and aspects of perfectionism). Of particular note, perfectionism, and its constituent dimensions, was one such construct expected to be inversely associated with mindfulness subscales based on the research of Hinterman, Burns, Hopwood, and Rogers (2012). Thienot and colleagues found in the development of the MIS measure a positive relationship between the MIS awareness subscale and personal standards perfectionism (PSP), yet a negative relationship between the MIS refocusing subscale and the evaluative concerns perfectionism (ECP). Perhaps then, the relationship between mindfulness and perfectionism depends upon the particular dimensions of the constructs under consideration, much like the relationship between perfectionism and sport performance. Future research examining the relationship between mindfulness and perfectionism in sport, specifically within a population of highly perfectionistic athletes, may therefore extend these findings. The sport-specific and performance-oriented language used throughout MIS items may on one hand make the measure well-suited to 
assessing mindfulness in sport, and on the other hand be somewhat less reflective of traditional tenets of mindfulness than other available instruments.

The Athletic Mindfulness Questionnaire (AMQ; Zhang, Chung, \& Si, 2017) is a 16-item, 3-factor sport-specific measure of mindfulness. Mindfulness is assessed in the current questionnaire based on the subscales present moment attention, awareness, and acceptance. Items are rated on 5-point Likert scales ranging from 1 (never true) to 5 (always true). Confirmatory factor analysis (CFA) revealed satisfactory fit indices for the 16-item, 3-factor structure of the instrument, $X^{2}(101)=221.28 \mathrm{p}<.001, \mathrm{CFI}=0.95, \mathrm{TLI}=0.94, \mathrm{WRMR}=1.04, \mathrm{RMSEA}=0.06$. Once AMQ items were directly worded rather than reverse-worded, convergent validity for the measure was supported through significant positive associations between the present moment attention, awareness, and acceptance subscales of the AMQ and mindfulness as measured by the MAAS. Concurrent validity for the three subscales of the AMQ was also supported through significant negative relationships with burnout and experiential avoidance, and significant positive relationships with well-being, positive affect, and dispositional flow. The single-factor structure of the model revealed a poor fit to the data, providing support for the multidimensional nature of mindfulness. Given that the measure was developed using samples of athletes from an Eastern country participating in a variety of team and individual sports, the authors suggest that future researchers consider validating the AMQ using athletes from Western countries to better understand the generalizability of the instrument. Zhang and colleagues also addressed in their measure potential limitations to the theoretical foundation of mindfulness as assessed in the MIS. For example, the authors note a potential shortcoming in that the MIS was developed to assess refocusing skills, and does not also consider attention sustaining skills. The AMQ may therefore capture important information regarding mindfulness skills both at sustaining attention (e.g., "I 
can easily sustain my attention on the competition) and bringing back attention (e.g., "When I find myself distracted, I gently bring my attention back to my training”). Further, the AMQ appears to assess mindfulness among athletes in a way that aligns more closely with traditional literature on facets of mindfulness - and domain-general measures of mindfulness - than other existing sport-specific measures. These features of the instrument may lend support for the use of the AMQ when exploring mindfulness in sport.

\section{Perfectionism}

Similar to the present state of measurement in mindfulness outlined previously, researchers have developed a variety of instruments to measure perfectionism, both general and specific to sport. A collection of well-known self-report measures of perfectionism are included in Table 2. Prominent frameworks discussed in the perfectionism literature have described perfectionism as a construct consisting of multiple dimensions or facets (Frost et al., 1990; Hewitt \& Flett, 1991). Researchers suggest that dimensions of perfectionism may be organized within two higher-order factors, namely perfectionistic strivings and perfectionistic concerns (Stoeber \& Otto, 2006), thus helping to integrate multi-facet conceptualizations of perfectionism. Although perfectionistic strivings and perfectionistic concerns are moderately to highly correlated, researchers have largely supported a two-factor conceptualization of perfectionism (e.g., Dunkley et al., 2012). Given the high degree of shared variance between the two higher order dimensions of perfectionism, however, perhaps a single general factor might explain the multidimensional perfectionism construct. Smith and Saklofske (2017) sought to examine the conceptual integration of these dimensions of perfectionism through confirmatory factor analysis (CFA) and structural equation modeling (SEM). Subscales drawn from the HFMPS, the FMPS, and the APS-R were used to assess alternate structural models of perfectionism, with 
perfectionistic strivings measured using four subscales (i.e., high standards, personal standards, self-oriented perfectionism, and order), and perfectionistic concerns measured using five subscales (i.e., socially prescribed perfectionism, other-oriented perfectionism, doubts about actions, concern over mistakes, and discrepancy). Upon evaluating both a two-factor model and a bifactor model with a general perfectionism factor, the latter was found to best represent the structure of perfectionism. The presence of a general factor that may explain common variance in the perfectionistic strivings and perfectionistic concerns dimensions speaks toward the complex nature of perfectionism as a measured construct, and supports additional efforts to seek further clarity regarding the factor structure and dimensionality of perfectionism.

Measurement of perfectionism has also been translated into the realm of sport. For instance, Haase and Prapavessis (2004) aimed to validate the factor structure of the PANPS for use with athlete participants. A sample of 540 male and female elite athletes from Australia and New Zealand across team and individual sports (i.e., aerobics, archery, basketball, cricket, cycling, diving, gymnastics, hockey, kayaking, netball, rowing, rugby, soccer, squash, softball, volleyball, water polo, and wrestling) completed the original 40-item, 2-factor (i.e., positive and negative perfectionism) PANPS. Items were rated on 5-point Likert scales from 1 (strongly disagree) to 5 (strongly agree). CFA for the original measurement model revealed an unsatisfactory fit to the data, $X^{2}=2295.29, \mathrm{p}<.001, X^{2} / d f=3.11, \mathrm{GFI}=0.80, \mathrm{NNFI}=0.62, \mathrm{CFI}$ $=0.71$, RMSEA $=0.06$; thus, the researchers next refined the factor structure of the PANPS through EFA and CFA. Results yielded a 19-item, 2-factor model measuring the positive and negative perfectionism constructs with a more satisfactory fit to the data, $X^{2}=345.96, \mathrm{p}<.001$, $X^{2} / d f=2.29, \mathrm{GFI}=0.93, \mathrm{NNFI}=0.87, \mathrm{CFI}=0.92, \mathrm{RMSEA}=0.05$. The reconstituted version of the PANPS may be a tenable abbreviated 2-factor measure of perfectionism in sport. Despite 
revealing initial support for the use of a revised PANPS for athletes, further research is needed to confirm the factor structure and establish the construct validity of the instrument.

In similar fashion, other researchers have aimed to bring a general framework of perfectionism into the context of sport. Hill, Appleton, and Mallinson (2016) conducted a series of studies to develop and initially validate a sport-specific multidimensional measure of performance perfectionism grounded in the three-facet conceptualization of perfectionism offered by Hewitt and Flett (1991). Due to the notion that performance is a defining feature of the sport context, the Performance Perfectionism Scale for Sport (PPS-S; Hill et al., 2016) is a 3factor measure assessing self-oriented, other-oriented, and socially prescribed performance perfectionism. Three samples of youth and young adult athletes from a range of individual and team sports were recruited to complete the initially developed 12-item, 3-factor PPS-S. The authors used both CFA and exploratory structural equation modeling (ESEM) to examine the factor structure of the scale, and examined construct validity based on associations between dimensions of the PPS-S and another highly regarded sport-specific perfectionism measure, the Sport-MPS-2. In two out of the three samples, CFA for the measurement model indicated an adequate fit to the data [e.g., $X^{2}(51)=114.99, \mathrm{CFI}=0.91, \mathrm{RMSEA}=0.07, \mathrm{SRMR}=0.06, \mathrm{AIC}=$ 9679.28, $\mathrm{BIC}=9813.54, \mathrm{ABIC}=9689.93]$. Standard parameter estimates from the three CFAs indicated significant, large factor loadings across all items. ESEMs corroborated the CFA findings with greater clarity, providing support for the 3-factor PPS-S model fit. Bivariate correlations revealed that the three subscales of the PPS-S were significantly and positively associated with all subscales of the Sport-MPS-2, corroborating the construct validity of the scale. The factor structure of the newly developed PPS-S appears to be adequately supported in alignment with the conceptualization of perfectionism posited by Hewitt and Flett (1991). 
Further validation of the measure is warranted, however, to generalize use of the PPS-S to adult athletes in other sport contexts.

The Perfectionism in Sport Scale (PSS; Anshel \& Eom, 2003) is a 32-item, 4-factor sport-specific measure of perfectionism developed based on psychometrically validated nonsport instruments of perfectionism (Frost et al., 1990; Howitt \& Flett, 1991). The measure was developed using a sample of moderate - highly skilled athletes $(N=384)$ with competitive sport experience within two years of the study, ranging in age from 17 to 47 years $(M=22.4, S D=$ 4.3). Participants were sampled from a university in the US, and represented sports including basketball, gymnastics, soccer, swimming, tennis, and track and field. The four key dimensions assessed in the scale include concern about mistakes, parental and coach criticism, and personal standards. The 4-factor structure of the instrument was established using EFA, with a factor loading criterion of .40 . The researchers demonstrated low intercorrelations between the four primary dimensions, supporting the independence of the measured constructs. Cronbach's alpha values ranging from .80 to .89 reflect satisfactory internal consistency of the scale.

The Multidimensional Inventory of Perfectionism in Sport (MIPS; Stoeber, Otto, \& Stoll, 2006; Madigan, 2016) is a 26-item, 4-factor domain specific measure of perfectionism. The primary dimensions assessed in the inventory include the subscales striving for perfection, negative reactions to imperfection, and parental and coach pressures to be perfect. Items are rated on 5-point Likert scales from 1 (strongly disagree) to 5 (strongly agree). CFA revealed that the 4-factor model of the measure provided a satisfactory fit to the data, $X^{2}(293)=1159.76, \mathrm{CFI}=$ $.91, \mathrm{TLI}=.90, \mathrm{RMSEA}=.08, \mathrm{SRMR}=.05$. The single factor model did not adequately fit the data, however, supporting perfectionism as a multidimensional construct. All items displayed acceptable internal consistency reliability, with Cronbach's alpha values ranging from .89-.96, 
and loaded significantly onto the appropriate factors, with factor loadings ranging from .71-.90 across items. The available psychometric data for the MIPS support the instrument as a valid and reliable measure of perfectionism in sport.

The Sport Multidimensional Perfectionism Scale (Sport-MPS; Dunn et al., 2002, 2006) and the updated Sport Multidimensional Perfectionism Sale-2 (Sport-MPS-2; Gotwals \& Dunn, 2009; Gotwals, Dunn, Causgrove Dunn, \& Gamache, 2010; Dunn et al., 2016) were derived from the Frost et al. (1990) conceptualization of perfectionism, and developed for use within the context of sport. The newer version of the scale will be discussed herein to present the most recent state of the measure. The Sport-MPS-2 is a 42-item, 6-factor (i.e., personal standards, concern over mistakes, perceived parental and coach pressures, doubts about actions, and organization) measure of perfectionism in sport. Items are rated on 5-point Likert scales, ranging from 1 (strongly disagree) to 5 (strongly agree). Findings the CFA indicated that the 6-factor model fit the data adequately $(\mathrm{RMSEA}=.06, \mathrm{RMSR}=.08, \mathrm{CFI}=.93)$. Due to the lack of multivariate normality in the data, GLS was used to corroborate the model fit. When the model was reanalyzed, however, the model structure did not provide a satisfactory fit to the data $(\mathrm{RMSEA}=.14, \mathrm{RMSR}=.13, \mathrm{CFI}=.75) . \mathrm{EFA}$ was then conducted to explore the factor structure of the measure. Results indicated that the latent dimensionality of the Sport-MPS-2 was represented adequately by the 6-factors, with three items showing potentially questionable factor loading patterns. The 6-factor structure was retained following this series of factor analyses. Given that the results of the current EFA (Gotwals et al., 2010) did not fully confirm the empirical and theoretical validity demonstrated previously (Gotwals \& Dunn, 2009), further research is needed to confirm the factor structure of the measure. As expected, moderate to strong positive relationships arose between the conceptually similar Sport-MPS-2 and Frost-MPS 
subscales, supporting the convergent validity of the measure. Furthermore, Dunn et al. (2016) examined the higher-order latent dimensionality of the Sport-MPS-2, revealing that the six subscales may serve as meaningful indicators of two higher-order factors - perfectionistic strivings and perfectionistic concerns. Thus, the Sport-MPS-2 may be used as a measure of the higher-order dimensionality of perfectionism, supporting the theoretical integration of multidimensional perfectionism frameworks and the notions of perfectionistic strivings and perfectionistic concerns. For a review of perfectionism measurement in sport, dance, and exercise, interested readers are directed to the work of Stoeber and Madigan (2016). The authors offer both critique and recommendations in their review of perfectionism measurement, and assert that both the Sport-MPS and the more recent Sport-MPS-2 are strong selections for measuring perfectionism in sport. Stoeber and Madigan also note in their discussion that some items may need adapting to suit the language of particular sport contexts. Taken together with recent literature using these measures to cluster athletes on facets of perfectionism, the SportMPS and Sport-MPS-2 may be well-suited to the study of perfectionism among athletes through self-report methods.

\section{Performance}

Enhancement of sport performance is often a primary goal of developed approaches in sport and performance psychology. The ability to assess the impact of a given approach on the performance of athletes is thus pivotal to our understanding of the efficacy of various approaches to performance enhancement. Measurement of performance in sport and performance psychology research has been carried out through numerous methods (e.g., subjective assessments, controlled settings, pre- and post-intervention, standings/rankings, etc.), however, 
assessing sport performance through real-world, objective measures has traditionally been noted in the literature as a key area of focus for future research efforts.

Such efforts to measure sport performance objectively have begun to expand. Case study research exploring the effectiveness of mindfulness training has integrated objective measures of performance in sports like power lifting (Gardner \& Moore, 2004) and figure skating (Bernier, Thienot, Pelosse, \& Fournier, 2014). In their one-year follow-up study regarding the athletic performance enhancing effects of the MSPE program, Thompson et al. (2011) acknowledged that comparison of athletes' best performance scores pre- and post-intervention may not have been an ideal way to measure changes in sport performance. The researchers recommended instead that future measurement of athletic performance include average scores, which may be a more valid assessment of performance in sport. Gooding and Gardner (2009) based their objective sport performance measure on competitive free throw attempts across the regular season in NCAA Division I male basketball players. Sport performance among basketball players was therefore able to be determined based on season-long performance as opposed to a best effort. Bernier et al. (2009) assessed performance improvements among golfers in a mindfulness-based program based on national ranking, a performance measure across time. John et al. (2011) measured sport performance in shooting based on shooting accuracy or shooting score, indicating a compilation of trials from which to examine performance improvements. In still further performance-focused research, Stoeber et al. (2009) employed triathlon times as an objective measure of athletic performance relative to perfectionistic tendencies. The authors justified their selection of this performance measure by indicating that, in an event like the triathlon, performance is to a large extent self-determined and relatively independent of others' performance outcomes. These individual performance elements may provide a rationale 
for the study of psychological constructs, like mindfulness and perfectionism, in sports where athletes' performance is highly self-determined and involve more closed- than open-skills. Van Dyke et al. (2018) studied self-talk use among a sample of collegiate gymnasts relative to realworld competitive performance outcomes. The researchers measured objective performance by calculating a season average balance beam score for each athlete, and measured performance consistency by calculating a standard deviation of balance beam scores from across the competition season for each athlete. Such a method has great utility for future research in sports that have objective and publicly available records of competitive performance. Performance in gymnastics, much like the triathlon performance studied by Stoeber et al. (2009), has the benefit of being largely self-determined and independent of others' performance levels. Thus, a sport like gymnastics may be particularly well-suited to the study of psychological constructs in relation to objective measures of individual performance outcomes. Despite recent growth in objective measurement of sport performance, still further research is needed to elucidate how phenomena like mindfulness and perfectionism influence the performance of high level athletes.

\section{Directions for Future Research}

When considering mindfulness, perfectionism, and performance together, the following links have been initially supported: (1) a negative relationship between mindfulness and maladaptive aspects of perfectionism, (2) a negative relationship between maladaptive perfectionism and performance, (3) a positive relationship between adaptive perfectionism and performance, and (4) a positive relationship between mindfulness and performance. Taken together, mindfulness-based approaches may have utility for both decreasing maladaptive perfectionism and facilitating performance among athletes. Such a link may indeed exist. For instance, Thompson et al. (2011) offered preliminary support indicating that athletes who engage 
in mindfulness-based practices may experience both enhanced performance and diminished maladaptive perfectionism. It is important to note that mindfulness and psychological skills are likely to have a particularly strong influence on performance when skill levels are high, and physical ability and skill technique do relatively little to differentiate the performance of athletes. The utility and effectiveness of mindfulness-based approaches may therefore be specific to the sport performance context. Thus, the type of performance and the level of skill among athletes must be taken into consideration in the development of future research directions.

The multifaceted relationships among these constructs may have unique relevance in certain sport contexts. For example, in a sport like gymnastics in which the quality of performance is judged by others and held to a standard of perfection, the study of mindfulness together with perfectionism and performance outcomes may have important implications for athletes' optimal sport experiences. The study of nonjudgmental acceptance, a key component of mindfulness, and perfectionism among athletes in a sport that quite overtly emphasizes being judged and striving for perfection seems particularly fitting. Therefore, judged and technically demanding sports may indeed be prime settings in which Flett and Hewitt's (2005) perfectionism paradox could surface among athletes.

Additionally, mindfulness-based approaches have often been distinguished from traditional PST approaches in that volitional efforts to control and regulate attention are not emphasized. In sports that require the performance of many complex skills in which attentional resources are key, mindfulness-based approaches may be quite effective - perhaps allowing athletes to devote fewer attentional resources to self-regulatory processes and thus conserve valuable attentional capacity for the sport task at hand. Understanding mindfulness, perfectionism, and how these constructs are associated with real-world competitive performance 
among high level athletes in sports requiring the execution of technically complex skills may therefore be a fitting extension of existing literature.

As evident in previous sections outlining measurement of mindfulness and perfectionism, there are numerous options from which to choose. Beyond the selection of domain-specific measures appropriate to the study context, future researchers might also consider the factor structure of the various measurement options to select those that best match the purpose of the study. For instance, researchers have used different conceptualizations of the multidimensional nature of perfectionism as the foundational theory upon which their measures of perfectionism were developed. Any study findings drawn from a particular self-report perfectionism measure will therefore necessarily be viewed through the lens of the underlying theoretical framework. In a call for greater attention toward the measurement of mindfulness, Van Dam et al. (2018) suggest that mindfulness may constitute a semantically ambiguous construct. The authors thus encourage future researchers using self-report measures to note the exact measure employed, and to further outline the specific aspects/factors of mindfulness assessed in the chosen measure.

Within the fields of psychological and other basic and applied sciences, researchers have highlighted the importance of replication efforts to support the integrity of published research findings (Van Dam et al., 2018). Instruments created to measure constructs of relevance to sport performance are developed using some selected athlete population, and may subsequently be used with another athlete population without confirming the psychometric properties of the instruments. It may therefore be prudent to perform such psychometric replication efforts prior to conducting subsequent analyses when the makeup of the athlete population of interest is different than that originally included in the development and validation of the instruments used.

Confirmatory Factor Analysis (CFA) has been considered the gold standard for examining the 
construct validity of a developed measure (Marchant, 2013). Prior to using a given measure in a new context, researchers have deemed CFA a necessary precursor to performing subsequent analyses.

Scholars have also noted the relative scarcity of person-centered research in comparison to variable-centered research in the field of sport and performance psychology (Gucciardi et al., 2012). Person-centered approaches allow the researcher to reveal unique profiles of individuals with different characteristics. To date, researchers have used person-centered approaches, in the form of cluster analyses, to better understand individual characteristics of perfectionism (e.g., Gucciardi et al., 2012) and mindfulness (e.g., Kee \& Wang, 2008) in sport. Such studies, however, are relatively few, and although mindfulness and perfectionism have been studied using cluster analytic approaches individually, to the researcher's knowledge the two constructs have yet to be combined in a single person-centered analysis. By exploring both mindfulness and perfectionism constructs simultaneously, particularly when looking at mindfulness characteristics of athletes in a highly perfectionistic sport, one might gain a deeper, more nuanced understanding of how the two are experienced together among high level athletes.

Additionally, linking psychological phenomena to real-world objective measures of performance in sport continues to be an area in need of further attention. For sports in which performance records are made publicly available across multiple seasons of competition, the study of key constructs as they relate to athletes' competitive performance becomes feasible. Such studies can contribute to filling the present gap within the literature regarding psychological constructs of interest, including mindfulness and perfectionism, and objective measures of sport performance. Future research efforts should aim to extend objectively measured performance findings to more varied sport contexts, and to further control for context 
variables related to individual performance (e.g., age, skill level, gender, etc.). By integrating objective measures of performance and context variable control into study designs, the rigor of future research linking psychological constructs to sport may be improved. Mindfulness-based intervention studies using objective performance measures as key outcome variables may be improved through comparison with a PST or psychoeducation standard (e.g., providing athletes in control groups with alternative tasks to compare effectiveness), and through control of potentially confounding variables that are closely related to intervention effectiveness (e.g., past exposure to constructs of interest).

As a concluding remark, the conceptual model described previously may help to inform the design of future research on various pathways of influence regarding mindfulness, perfectionism, and performance. How might mindfulness and perfectionism be experienced together among high level individual athletes in a judged sport? How might athletes who experience differences in mindfulness and perfectionism differ in adaptive performance outcomes in real-world competitive contexts? What is the strength of the link between both mindfulness and perfectionism, and performance in sport? These are just a few such questions that might be explored to draw from and extend the current state of empirical literature pertaining to mindfulness, perfectionism, and performance among competitive athletes. Illuminating answers to these and similar questions might have positive implications for the work of coaches, sport psychology practitioners, and researchers. 


\section{References}

Aherne, C., Moran, A.P., \& Lonsdale, C. (2011). The effect of mindfulness training on athletes' flow: An initial investigation. The Sport Psychologist, 25, 177-189.

Andersen, M. B., McCullagh, P., \& Wilson, G. (2007). But what do the numbers really tell us? Arbitrary metrics and effect size reporting in sport psychology research. Journal of Sport \& Exercise Psychology, 29, 664-672.

Andersen, M. B., \& Waterson, A. K. (2017). A brief impressionistic history of paying attention: The roots of mindfulness. In S. J. Zizzi \& M. B. Andersen (Eds.), Being mindful in sport and exercise psychology: Pathways for practitioners and students (pp. 15-27). Morgantown, WV: FiT Publishing.

Anshel, M. H., \& Eom, H.-J. (2003). Exploring the dimensions of perfectionism in sport. International Journal of Sport Psychology, 34, 255-271.

Baer, R. A., Smith, G. T., \& Allen, K. B. (2004). Assessment of mindfulness by self-report: The Kentucky inventory of mindfulness skills. Assessment, 11, 191-191.

Baer, R. A., Smith, G. T., Lykins, E., Button, D., Krietemeyer, J., Sauer, S., Walsh, E., Duggan, D., \& Williams, J. M. G. (2008). Construct validity of the Five Facet Mindfulness Questionnaire in meditating and nonmeditating samples. Assessment, 15, 329-342.

Baltzell, A. L., \& Diehl, R. (2017). Flow theory: Applications to mindfulness and sport. In S. J. Zizzi \& M. B. Andersen (Eds.), Being mindful in sport and exercise psychology: Pathways for practitioners and students (pp. 133-153). Morgantown, WV: FiT Publishing.

Baltzell, A., \& Summers, J. (2017). The power of mindfulness: Mindfulness meditation training in sport (MMTS). New York, NY: Springer International Publishing. 
Batten, S. (2011). Essentials of acceptance and commitment therapy. Thousand Oaks, CA: Sage.

Bergomi, C., Tschacher, W., \& Kupper, Z. (2013). The assessment of mindfulness with selfReport measures: Existing scales and open issues. Mindfulness, 4(3), 191-202. doi:10.1007/s12671-012-0110-9

Bernier, M., Thienot, E., Codron, R., \& Fournier, J. F. (2009). Mindfulness and acceptance approaches in sport performance. Journal of Clinical Sports Psychology, 4, 320-333.

Bernier, M., Thienot, E., Pelosse, E., \& Fournier, J. F. (2014). Effects and Underlying Processes of a Mindfulness-Based Intervention with Young Elite Figure Skaters: Two Case Studies. Sport Psychologist, 28(3), 302-315.

Birrer, D., Röthlin, P., \& Morgan, G. (2012). Mindfulness to enhance athletic performance: Theoretical considerations and possible impact mechanisms. Mindfulness, 3, 235-246. https://doi.org/10.1007/s12671-012-0109-2

Bodner, T., \& Langer, E. (2001). Individual differences in mindfulness: The Langer Mindfulness Scale. Poster session presented at the annual meeting of the American Psychological Society, Toronto, Ont., Canada.

Brown, K.W., \& Ryan, R.M. (2003). The benefits of being present: Mindfulness and its role in psychological well-being. Journal of Personality and Social Psychology, 84, 822-848.

Bühlmayer, L. Birrer, D., Röthlin, P., Faude, O., \& Donath, L. (2017). Effects of mindfulness practice on performance-relevant parameters and performance outcomes in sports: A meta-analytical review. Sports Medicine, 47, 2309-2321. doi: 10.1007//s40279-017-07529 
Cardaciotto, L., Herbert, J. D., Forman, E. M., Moitra, E., \& Farrow, V. (2008). The assessment of present-moment awareness and acceptance the Philadelphia mindfulness scale. Assessment, 15, $204-223$.

Conroy, D.E., Elliot, A.J., \& Hofer, S.M. (2003). A 2 x 2 achievement goals questionnaire for sport: Evidence for factorial invariance, temporal stability, and external validity. Journal of Sport \& Exercise Psychology, 25, 456-476.

Csikszentmihalyi. M. (1990). Flow: The psychology of optimal experience. New York: Harper \& Row.

De Petrillo, L.A., Kaufman, K.A., Glass, C.R., \& Arnkoff, D.B. (2009). Mindfulness for longdistance runners: An open trial using mindful sport performance enhancement (MSPE). Journal of Clinical Sport Psychology, 4, 357-376.

Dimidjian, S., Kleiber, B. V., \& Segal, Z. V. (2009). Mindfulness-based cognitive therapy. In M. A. K. N. D. Reinecke (Ed.), Cognitive and behavioral theories in clinical practice (307 331). New York, NY: Guilford Press.

Dunkley, D. M., Blankstein, K. R., \& Berg, J. L. (2012). Perfectionism dimensions and the fivefactor model of personality. European Journal of Personality, 26, 233-244. doi: $10.1002 /$ per.829

Dunn, J. G. H., Causgrove Dunn, J., Gotwals, J. K., Vallance, J. K. H., Craft, J. M., \& Syrotuik D. G. (2006). Establishing construct validity evidence for the sport multidimensional perfectionism scale. Psychology of Sport and Exercise, 7, 57 - 79.

Dunn, J.G.H., Causgrove Dunn, J., \& Syrotuik, D.G. (2002). Relationship between multidimensional perfectionism and goal orientation in sport. Journal of Sport \& Exercise Psychology, 24, 376-395. 
Dunn, J., Gotwals, J., Causgrove Dunn, J., Selzler, A., Lizmore, M., Vaartstra, M., . . Gamache, V. (2016). A multi-sample investigation of the higher-order latent dimensionality of the sport-multidimensional perfectionism scale-2. Psychology of Sport \& Exercise, 27, 150156. doi:10.1016/j.psychsport.2016.08.006

Flett, G., \& Hewitt, P. (2005). The perils of perfectionism in sports and exercise. Current Directions in Psychological Science, 14, 14-18. doi:10.1111/j.0963-7214.2005.00326.x

Frost, R. O., Marten, P., Lahart, C., \& Rosenblate, R. (1990). The dimensions of perfectionism. Cognitive Therapy and Research, 14, 449-468.

Gardner, F., \& Moore, Z. (2004). A mindfulness-acceptance-commitment-based approach to athletic performance enhancement: Theoretical considerations. Behavior Therapy, 35, $707-723$.

Gardner, F. L., \& Moore, Z. E. (2006). Clinical sport psychology. Champagne, IL: Human Kinetics.

Gardner, F. L., \& Moore, Z. E. (2007). The psychology of enhancing human performance: The mindfulness-acceptance-commitment (MAC) approach. New York, NY: Random House.

Gardner, F. L., \& Moore, Z. E. (2012). Mindfulness and acceptance models in sport psychology: A decade of basic and applied scientific advancements. Canadian Psychology, 53, 309318.

Gaudreau, P., \& Thompson, A. (2010). Testing a 2 x 2 model of dispositional perfectionism. Personality and Individual Differences, 48, 532-537.

Gigerenzer, G. (2004). Mindless statistics. Journal of Socio-Economics, 33, 587-606. http://dx.doi.org/10.1016/j.socec.2004.09.033 
Gooding, A., \& Gardner, F. (2009). An investigation of the relationship between mindfulness, preshot routine, and basketball free throw percentage. Journal of Clinical Sport Psychology, 3(4), 303-319. doi:10.1123/jcsp.3.4.303

Gotwals, J.K. (2011).Perfectionism and burnout within intercollegiate sport: A person-oriented approach. The Sport Psychologist, 25, 489-510.

Gotwals, J. K., \& Dunn, J. G. H. (2009). A multi-method multi-analytic approach to establishing internal construct validity evidence: The sport multidimensional perfectionism scale 2 . Measurement in Physical Education and Exercise Science, 13, 71 - 92.

Gotwals, J. K., Dunn, J. G. H., Causgrove Dunn, J., \& Gamache, B. (2010). Establishing validity evidence for the sport multidimensional perfectionism scale-2 in intercollegiate sport. Psychology of Sport and Exercise, 11, 423 - 432.

Gotwals, J. K., Stoeber, J., Dunn, J. G. H., \& Stoll, O. (2012). Are perfectionistic strivings in sport adaptive? A systematic review of confirmatory, contradictory, and mixed evidence. Canadian Psychology, 53(4), 263-279. DOI 10.1037/a0030288

Gucciardi, D. F., Mahoney, J., Jalleh, G., Donovan, R. J., \& Parkes, J. (2012). Perfectionistic profiles among elite athletes and differences in their motivational orientations. Journal of Sport \& Exercise Psychology, 34, 159-183.

Haase, A. M., \& Prapavessis, H. (2004). Assessing the factor structure and composition of the Positive and Negative Perfectionism Scale in sport. Personality and Individual Differences, 36(7), 1725-1740. http://dx.doi.org/10.1016/j.paid.2003.07.013

Hair, J. F., Jr., Anderson, R. E., Tatham, R. L., \& Black, W. C. (1998). Multivariate data analysis. Englewood Cliffs, NJ: Prentice-Hill. 
Hardy, L., Jones, J., \& Gould, D. (1996). Understanding psychological preparation for sport: Theory and practice of elite performers. Chichester: Wiley.

Hatzigeorgiadis, A., \& Biddle, S.J.H. (2000). Assessing cognitive interference in sport:

Development of the Thought Occurrence Questionnaire for Sport. Anxiety, Stress, and Coping, 13, 65-86.

Hayes, S. C., Strosahl, K., \& Wilson, K. G. (1999). Acceptance and commitment therapy: An experiential approach to behavior change. New York, NY: Guilford Press.

Hewitt, P. L., \& Flett, G. L. (1991). Perfectionism in the self and social contexts: Conceptualization, assessment, and association with psychopathology. Journal of Personality and Social Psychology, 60(3), 456-470. http://dx.doi.org/10.1037/00223514.60.3.456

Hill, A. P., Appleton, P. R., \& Mallinson, S. H. (2016). Development and initial validation of the Performance Perfectionism Scale for Sport (PPS-S). Journal of Psychoeducational Assessment, 34, 653-669. https://doi.org/10.1177/0734282916651354

Hill, A. P., Mallinson-Howard, S. H., \& Jowett, G. E. (2018). Multidimensional perfectionism in sport: A meta-analytical review. Sport, Exercise, and Performance Psychology, 7(3), 235-270. http://dx.doi.org/10.1037/spy0000125

Hinterman, C., Burns, L., Hopwood, D., \& Rogers, W. (2012). Mindfulness: Seeking a more perfect approach to coping with life's challenge. Mindfulness, 3, 275-281. http://dx.doi.org/10.1007/s12671-012-0091-8.

Ivarsson, A., Johnson, U., Andersen, M. B., Fallby, J., \& Altemyr, M. (2015). It pays to pay attention: A mindfulness-based program for injury prevention with soccer players. 
Journal of Applied Sport Psychology, 27, 319 - 334. doi:

$10.1080 / 10413200.2015 .1008072$

Jackson, S. A., \& Csikszentmihalyi, M. (1999). Flow in sports: The key to optimal experience and performances. Champaign, IL: Human Kinetics.

Jackson, S.A., \& Eklund, R.C. (2002). Assessing flow in physical activity: The Flow State Scale2 and Dispositional Flow Scale-2. Journal of Sport \& Exercise Psychology, 24, 133-150.

Jackson, S. A., \& Eklund, R. C. (2004). The flow scale manual. Morgantown, WV: Fitness Information Technology.

John. S., Verma, S.K., \& Khanna, G.L. (2011). The effect of mindfulness meditation on HPAaxis in pre-competition stress in sports performance of elite shooters. National Journal of Integrated Research in Medicine, 2, 15-21.

Kabat-Zinn, J. (1982). An outpatient program in behavioral medicine for chronic pain patients based on the practice of mindfulness meditation: Theoretical considerations and preliminary results. General Hospital Psychiatry, 4, 33-47.

http://dx.doi.org/10.1016/0163-8343(82)90026-3

Kabat-Zinn, J. (1990). Full catastrophe living: Using the wisdom of your body and mind to face stress, pain, and illness. New York: Dell Publishing.

Kabat-Zinn, J. (1994). Wherever you go, there you are: Mindfulness meditation in everyday life (1st ed.). New York, NY: Hyperion.

Kaufman, K. A., Glass, C. R., \& Arnkoff, D. B. (2009). Evaluation of Mindful Sport Performance Enhancement (MSPE): A new approach to promote flow in athletes. Journal of Clinical Sports Psychology, 4, 334-356. 
Kaufman, K. A., Glass, C. R., \& Pineau, T. R. (2017). Mindful sport performance enhancement: Mental training for athletes and coaches. Washington, DC: American Psychological Association.

Kee, Y. H., \& Wang, C. K. J. (2008). Relationships between mindfulness, flow dispositions and mental skills adoption: A cluster analytic approach. Psychology of Sport \& Exercise, 9(4) 393-411.

Kruschke, J. K. (2013). Bayesian estimation supersedes the $t$ test. Journal of Experimental Psychology: General, 142, 573-603. http://dx.doi.org/10.1037/a0029146

Langer, E. J. (2000). Mindful learning. Current Directions in Psychological Science, 9, 220-223.

Lau, M.A., Bishop, S.R., Segal, Z.V., Buis, T., Anderson, N.D., Carlson, L., et al. (2006). The Toronto Mindfulness Scale: Development and validation. Journal of Clinical Psychology, $62,1445-1467$.

Linehan, M. (1993). Cognitive-behavioral treatment of borderline personality disorder. New York: NY: Guilford Press.

Madigan, D. J. (2016). Confirmatory factor analysis of the multidimensional inventory of perfectionism in sport. Psychology of Sport and Exercise, 26, 48 - 51.

Marchant, D. B. (2013). Psychological assessment: Objective/self-report measures. In S. J. Hanrahan, \& M. B. Andersen (Eds.), Routledge handbook of applied sport psychology: A comprehensive guide for students and practitioners (pp. 111-119). New York, NY: Routledge.

Martens, R., Vealey, R., \& Burton, D. (1990). Competitive anxiety in sport. Champaign, IL: Human Kinetics. 
Martinent, G., \& Ferrand, C. (2006). A cluster analysis of perfectionism among competitive athletes. Psychological Reports, 99, 723-738.

McAlarnen, M. M., \& Longshore, K. (2017). Evidence-based mindfulness: Proceed, with caution. In S. J. Zizzi \& M. B. Andersen (Eds.), Being mindful in sport and exercise psychology: Pathways for practitioners and students (pp. 29-53). Morgantown, WV: FiT Publishing.

Meichenbaum, D. (1977). Cognitive-behavior modification: An integrative approach. New York: Plenum. http://dx.doi.org/10.1007/978-1-4757-9739-8

Moghadam, M.S.. Sayadi. E.. Samimifar. M.. \& Moharer, A. (2013). Impact assessment of mindfulness techniques education on anxiety and sports performance in Badminton players Isfahan. International Research Journal of Applied and Basic Sciences, 4, 1170 1175.

Noetel, M., Ciarrochi, J., Van Zanden, B., \& Lonsdale, C. (2017). Mindfulness and acceptance approaches to sporting performance enhancement: A systematic review. International Review of Sport and Exercise Psychology, doi:10.1080/1750984X.2017.1387803

Pineau, T., Glass, C., \& Kaufman, K. (2014). Mindfulness in sport performance. In A. Ie, C. T. Ngnoumen, \& E. J. Langer (Eds.), The Wiley Blackwell Handbook of Mindfulness (pp. 1004-1033). Chichester, UK: John Wiley \& Sons, Ltd. doi:10.1002/9781118294895.ch52

Rasquinha, A., Dunn, J. G. H., \& Causgrove Dunn, J. (2014). Relationships between perfectionistic strivings, perfectionistic concerns, and competitive sport level. Psychology of Sport and Exercise, 15, $659-667$.

Sapieja, K.M., Dunn, J.G.H., \& Holt, N.L. (2011). Perfectionism and perceptions of parenting styles in male youth soccer. Journal of Sport \& Exercise Psychology, 33, 20-39. 
Sappington, R., \& Longshore, K. (2015). Systematically reviewing the efficacy of mindfulnessbased interventions for enhanced athletic performance. Journal of Clinical Sport Psychology, 9, 232-262.

Sauer, S., Walach, H., Schmidt, S., Hinterberger, T., Lynch, S., Büssing, A., \& Kohls, N. (2013). Assessment of mindfulness: Review on state of the art. Mindfulness, 4(1), 3-17. doi:10.1007/s12671-012-0122-5

Segal, Z. V., Williams, J. M. G., \& Teasdale, J. D. (2002). Mindfulness-based cognitive therapy for depression: A new approach to preventing relapse. New York, NY: Guilford Press.

Smith, M. M., \& Saklofske, D. H. (2017). The structure of multidimensional perfectionism: Support for a bifactor model with a dominant general factor. Journal of Personality Assessment, 99, 297 - 303.

Smith, R.E., Smoll, F.L., \& Schutz, R.W. (1990). Measurement and correlates of sport-specific cognitive and somatic trait anxiety: The Sport Anxiety Scale. Anxiety Research, 2, 263280.

Stoeber, J. (2012). Perfectionism and performance. In S. M. Murphy (Ed.), The oxford handbook of sport and performance psychology (pp. 294-307). New York, NY: Oxford University Press. doi:10.1093/oxfordhb/9780199731763.013.0015

Stoeber, J. (2014). Perfectionism in sport and dance: A double-edged sword. International Journal of Sport Psychology, 45, 385-394. doi: 10.7352/IJSP 2014.45.385

Stoeber, J., \& Madigan, D. J. (2016). Measuring perfectionism in sport, dance, and exercise: Review, critique, recommendations. In A. P. Hill (Ed.), The psychology of perfectionism in sport, dance, and exercise (pp. 31-56). London: Routledge. 
Stoeber, J., \& Otto, K. (2006). Positive conceptions of perfectionism: Approaches, evidence, challenges. Personality and Social Psychology Review, 10, 295-319. doi:10.1207/s15327957pspr1004_2

Stoeber, J., Otto, K., \& Stoll, O. (2006). MIPS: Multidimensional Inventory of Perfectionism in Sport (English version, November 2006). School of Psychology, University of Kent. Available from https://kar.kent.ac.uk/41560/

Stoeber, J., Uphill, M. A., \& Hotham, S. (2009). Predicting race performance in triathlon: The role of perfectionism, achievement goals, and personal goal setting. Journal of Sport \& Exercise Psychology, 31, 211-245.

Teasdale, J. D., Segal, Z. V., Williams, J. M., Ridgeway, V. A., Soulsby, J. M., \& Lau, M. A. (2000). Prevention of relapse/recurrence in major depression by mindfulness-based cognitive therapy. Journal of Consulting and Clinical Psychology, 69(4), 615-623. doi: 10.1037//0022-006X.68.4.615

Thienot, E., Jackson, B., Dimmock, J., Grove, J. R., Bernier, M., \& Fournier, J. F. (2014). Development and preliminary validation of the mindfulness inventory for sport. Psychology of Sport and Exercise, 15, 72-80.

Thomas, P. R., Murphy, S. M., \& Hardy, L. (1999). Test of performance strategies: Development and preliminary validation of a comprehensive measure of athletes' psychological skills. Journal of Sports Sciences, 17, 697-711.

Thompson, R. W., Kaufman, K. A., De Petrillo, L. A., Glass, C. R., \& Arnkoff, D. B. (2011). One Year Follow-Up of Mindful Sport Performance Enhancement (MSPE) With Archers, Golfers, and Runners. Journal of Clinical Sport Psychology, 5(2), 99-116. 
Vallance, J.K.H., Dunn, J.G.H., \& Causgrove Dunn, J.L. (2006). Perfectionism, anger, and situation criticality in competitive youth ice hockey. Journal of Sport \& Exercise Psychology, 28, 383-406.

Van Dam, N., van Vugt, M., Vago, D., Schmalzl, L., Saron, C., Olendzki, A., . . Meyer, D. (2018). Mind the hype: A critical evaluation and prescriptive agenda for research on mindfulness and meditation. Perspectives on Psychological Science, 13, 36-61. doi:10.1177/1745691617709589

Van Dyke, E., Van Raalte, J., Mullin, E., \& Brewer, B. (2018). Self-Talk and competitive balance beam performance. The Sport Psychologist, 1-30, 1-30. doi:10.1123/tsp.20160085

Wilkinson, M. (2014). Distinguishing between statistical significance and practical/clinical meaningfulness using statistical inference. Sports Medicine, 44, 295-301. http://dx.doi.org/10.1007/s40279-013-0125-y

Wolanin, A. T., \& Gross, M. B. (2016). Mindfulness- and acceptance-based approaches with college student-athletes. In J. Block-Lerner, \& L. Cardaciotto (Eds.), The mindfulnessinformed educator: Building acceptance and psychological flexibility in higher education (pp. 155-172). New York, NY: Routledge.

Zhang, C., Chung, P., \& Si, G. (2017). Assessing acceptance in mindfulness with direct-worded items: The development and initial validation of the athlete mindfulness questionnaire. Journal of Sport and Health Science, 6, 311-320. doi:10.1016/j.jshs.2015.09.010 
Zizzi, S. J. (2017). Core concepts of mindfulness. In S. J. Zizzi \& M. B. Andersen (Eds.), Being mindful in sport and exercise psychology: Pathways for practitioners and students (pp. 314). Morgantown, WV: FiT Publishing. 
Table 1. Mindfulness Measurements

\section{Domain General Measures}

Cognitive and Affective Mindfulness Scale Revised (CAMS-R; Feldman, Hayes,

Kumar, Greeson, \& Laurenceau, 2007)

Five Factors Mindfulness Questionnaire (FFMQ; Baer et al., 2008)

Freiburg Mindfulness Inventory (FMI; Buchheld, Grossman, \& Walach, 2001; Walach,

Buchheld, Buttenmuller, Kleinknecht, \& Schmidt, 2006)

Kentucky Inventory of Mindfulness Skills (KIMS; Baer, Smith, \& Allen, 2004)

Mindful Attention Awareness Scale (MAAS; Brown \& Ryan, 2003)

Philadelphia Mindfulness Scale (PHLMS; Cardaciotto, Herbert, Forman, Moitra, \&

Farrow, 2008)

Southampton Mindfulness Scale (SMQ; Chadwick et al., 2008)

Toronto Mindfulness Scale (TMS; Lau et al., 2006)

Developmental Mindfulness Survey (DMS; Salloway \& Fischer, 2007)

Effects of Meditation Scale (EOM; Reavley \& Pallant, 2009)

Langer Mindfulness/Mindlessness Scale (MMS; Bodner \& Langer, 2001; Haigh,

Moore, Kashdan, \& Fresco, 2011)

\section{Sport-Specific Measures}

Athlete Mindfulness Questionnaire (AMQ; Zhang, Chung, \& Si, 2017)

Mindfulness Inventory for Sport (MIS; Thienot et al., 2014) 
Table 2. Perfectionism Measurements

\section{Domain General Measures}

Frost Multidimensional Perfectionism Scale (FMPS; Frost et al., 1990)

Hewitt and Flett Multidimensional Perfectionism Scale (HFMPS; Hewitt \& Flett, 1991)

Positive and Negative Perfectionism Scale (PANPS; Terry-Short, Owens, Slade, \&

Dewey, 1995)

Almost Perfect Scale-Revised (APS-R; Slaney, Rice, Mobley, Trippi, \& Ashby, 2001)

Perfectionism Inventory (PI; Hill et al., 2004)

\section{Sport-Specific Measures}

Perfectionism in Sport Scale (PSS; Anshel \& Eom, 2003)

Multidimensional Inventory of Perfectionism in Sport (MIPS; Stoeber, Otto, \& Stoll, 2006)

Sport Multidimensional Perfectionism Scale (Sport-MPS; Dunn et al., 2002, 2006)

Sport Multidimensional Perfectionism Scale-2 (Sport-MPS-2; Gotwals \& Dunn, 2009;

Gotwals, Dunn, Causgrove Dunn, \& Gamache, 2010) 
Table 3. Adapted Mindfulness-Flow-Performance Model in Sport

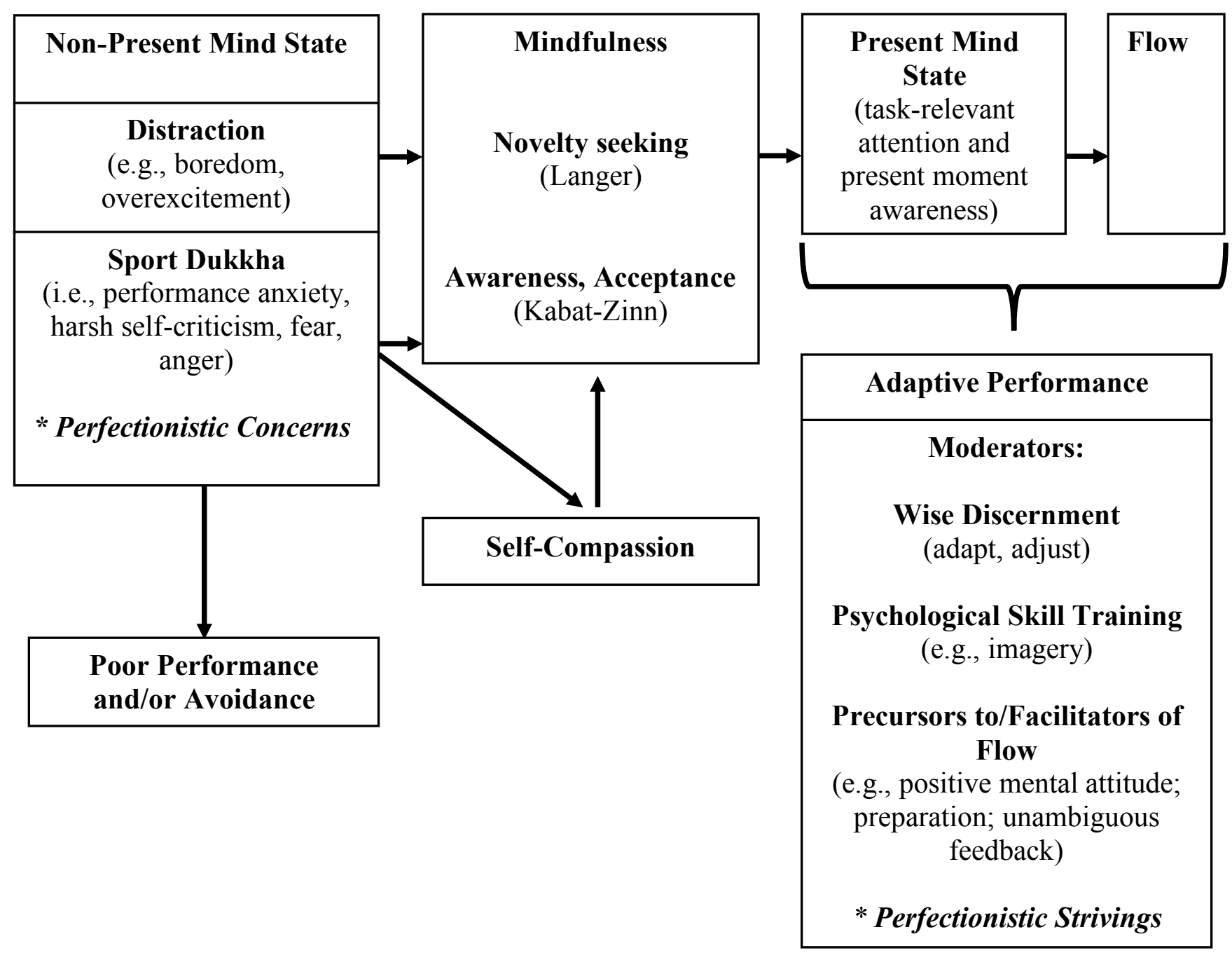

Figure adapted from the Mindfulness-Flow-Performance Model in Sport proposed by Baltzell and Diehl (2017). The italicized font and asterisks (*) indicate where dimensions of perfectionism may conceptually fit within the model. 


\section{Appendix A}

Athlete Mindfulness Questionnaire (AMQ; Zhang, Chung, \& Si, 2017)

INSTRUCTIONS The following statements relate to your general experiences in training and competition. Please indicate how much you agree or disagree with each statement based on your true feelings. Remember, there are no right or wrong answers, and just be honest. Please use the following scale to make your choice.

1. I can maintain my attention on my training.

\begin{tabular}{|c|c|c|c|}
\hline $\begin{array}{c}\text { Never } \\
\text { True }\end{array}$ & $\begin{array}{c}\text { Rarely } \\
\text { True }\end{array}$ & Sometimes & $\begin{array}{l}\text { Often } \\
\text { True }\end{array}$ \\
\hline
\end{tabular}

2. During training and competition, I can put up with

unpleasant thoughts and feelings.

3. I am aware that my emotions during training and competition can influence my thinking and behavior.

4. When something unexpected happens during training or competition, I am aware of my emotion state.

5. When I find myself distracted, I gently bring my attention back to my training.

6. When something during training or competition doesn't go well, I am aware of my inner frustration and restlessness.

7. During training and competition, it doesn't matter if the situation is good or bad, I can accept myself for who I am.

8. During training and competition, I can let go of the emotions brought about by negative life events.

9. I can easily sustain my attention on the competition.

10. When I feel muscular pain during training, I can still maintain attention on things I should do.

11. When the situation changes during the competition, I am aware of the thoughts and ideas that flashed across my mind.

12. When the competition process is totally beyond my expectations, I am aware of my physical reactions and changes.

13. During training and competition, it doesn't matter if my thoughts and feelings are comfortable or not, I put up with all of them.

14. If I notice that my mind is wandering, I can quickly get back to focusing on my training or competition

12

$\begin{array}{lll}3 & 4 & 5\end{array}$

$2 \quad 3 \quad 4 \quad 5$

$\begin{array}{lllll}1 & 2 & 3 & 4 & 5\end{array}$

$\begin{array}{lllll}1 & 2 & 3 & 4 & 5 \\ 1 & 2 & 3 & 4 & 5\end{array}$

$\begin{array}{lllll}1 & 2 & 3 & 4 & 5\end{array}$

$\begin{array}{lllll}1 & 2 & 3 & 4 & 5\end{array}$

$\begin{array}{lllll}1 & 2 & 3 & 4 & 5\end{array}$

$\begin{array}{lllll}1 & 2 & 3 & 4 & 5 \\ 1 & 2 & 3 & 4 & 5 \\ 1 & 2 & 3 & 4 & 5\end{array}$

$\begin{array}{llll}1 & 2 & 3 & 4\end{array}$

$\begin{array}{lllll}1 & 2 & 3 & 4 & 5\end{array}$

1

2

3

4

5 
15. During training or competition, I can be immediately

1

2

3

4

5 aware of my emotional changes.

16. Even though some thoughts and feelings during 1 2 3 training and competition may be unpleasant or miserable, I can get along with them peacefully. 


\section{Appendix B}

Sport Multidimensional Perfectionism Scale-2 (Sport-MPS-2; Gotwals \& Dunn, 2009)

INSTRUCTIONS The purpose of this questionnaire is to identify how athletes view certain aspects of their competitive experiences in sport. Please help us to more fully understand how gymnasts view a variety of their competitive experiences by indicating the extent to which you agree or disagree with the following statements. (Choose one response option to the right of each statement). Some of the questions relate to your sport experiences in general, while others relate specifically to experiences on the team that you have most recently competed with. There are no right or wrong answers so please don't spend too much time on any one statement; simply choose the answer that best describes how you view each statement.

\section{To what extent do you agree or disagree with the} following statements?

\section{Strongly \\ Disagree}

\section{Neither}

Agree nor

Disagree Disagree Agree Agree

Strongly

1. If I do not set the highest standards for myself in my sport, I am likely to end up a second-rate athlete.

2. Even if I fail slightly in competition, for me, it is as bad as being a complete failure.

3. I hate being less than the best at things in my sport.

4. If I fail in competition, I feel like a failure as a

\section{1}

1

(1)

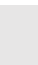
person.

5. The fewer mistakes I make in competition, the more people will like me.

6. It is important to me that I be thoroughly competent in everything I do in my sport.

7. I think I expect higher performance and greater results in my daily sport-training than most athletes.

8. I feel that other athletes generally accept lower standards for themselves in sport than I do.

9. I should be upset if I make a mistake in competition.

10. If a teammate or opponent performs better than me during competition, then I feel like I failed to some degree.

11. If I do not do well all the time in competition, I feel that people will not respect me as an athlete.

12. I have extremely high goals for myself in my sport.

13. I set higher achievement goals than most athletes who compete in my sport.

$\begin{array}{lllll}1 & 2 & 3 & 4 & 5 \\ 1 & 2 & 3 & 4 & 5 \\ 1 & 2 & 3 & 4 & 5\end{array}$
1

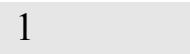

2

2

2

3

4

5

1

$\begin{array}{llll}2 & 3 & 4 & 5 \\ 2 & 3 & 4 & 5\end{array}$
5

1

2

3

4

5 
14. People will probably think less of me if I make mistakes in competition.

15. If I perform well but only make one obvious mistake in the entire competition, I still feel disappointed with my performance.

\begin{tabular}{lllll}
1 & 2 & 3 & 4 & 5 \\
1 & 2 & 3 & 4 & 5 \\
\hline
\end{tabular}


Appendix C

\section{Demographic Questionnaire}

Name:

Date:

Age:

College/University:

Highest level in gymnastics attained before college:
O 7
O 9
O Elite
O 8
O 10
O Other

Ethnicity:

O I am not Spanish, Hispanic, or Latino

O Cuban

O Mexican

O Cuban-American

O Mexican-American

O Some other Spanish, Hispanic, or

Latino group

O Chicano

O From multiple Spanish, Hispanic, or

Latino groups

O Puerto Rican

Race:

O White

O Native Hawaiian or other Pacific

Islander
O Black or African American
O From multiple races
O Asian
O Some other race
O American Indian or Alaska Native

Do you have previous experience working with a sport psychology professional (individually and/or in a team setting)?

O Yes, both individually and in a team setting

O Yes, individually

O Yes, in a team setting

O No 
If yes, how long have you worked with a sport psychology professional individually?

(Provide duration in months. Indicate 0 if you have not worked with a sport psychology professional individually)

If yes, how long have you worked with a sport psychology professional in a team setting?

(Provide duration in months. Indicate 0 if you have not worked with a sport psychology professional in a team setting)

Overall, how satisfied are you with the work you have done with a sport psychology professional?

O Extremely satisfied

O Somewhat dissatisfied

O Somewhat satisfied

O Extremely dissatisfied

O Neither satisfied nor dissatisfied

Do you have previous experience practicing mindfulness (e.g., meditation, Hatha yoga, formal classes/programs)?

O Yes

$\mathrm{O} \quad \mathrm{No}$

If yes, how long have you practiced mindfulness? (Duration in months)

If yes, describe your mindfulness experience(s): 


\section{Appendix D}

Email to Gymnastics Coaches Requesting Participants for a Research Project

Subject: Gymnast Participants Requested

Hello Coach

My name is Erika Van Dyke, and I am a former assistant women's gymnastics coach and current doctoral student in sport, exercise, and performance psychology. I am writing to ask for your athletes' participation in my research project. I would greatly appreciate your support in this important endeavor to learn more about how mindfulness and perfectionism influence competitive performance in gymnastics.

The study will involve completing a survey, expected to take approximately five minutes of their time. If your athletes would be willing to participate, I can mail the surveys to you with a return envelope enclosed or email a link to the online version of the survey.

Please feel free to contact me with any questions or comments and I will be happy to address them. Thank you very much.

Best regards,

Erika Van Dyke

Phone: XXX-XXX-XXXX

Email: XXXXXXX@XXX.XXX.edu 


\section{Appendix E}

Cover Letter and Consent to Participate Voluntarily in a Research Investigation Dear Participant,

This letter is a request for you to take part in a research project to explore experiences of mindfulness and perfectionism in gymnastics. This project is being conducted by Erika Van Dyke, MS in the College of Physical Activity and Sport Sciences at WVU with supervision of Dr. Sam Zizzi, a professor in the Department of Sport Sciences, for a Doctoral Degree in Sport Exercise and Performance Psychology. Your participation in this project is greatly appreciated and will consist of completing a short survey.

Your involvement in this project will be kept as confidential as legally possible. All data will be reported in the aggregate. You must be 18 years of age or older to participate. Identifiable information will be asked solely for the purpose of accessing gymnastics performance results, and this information will be stored in a manner that should not lead back to your identity as a participant. Your participation is completely voluntary. You may skip any question that you do not wish to answer and you may discontinue at any time. Your class and team standing will not be affected if you decide either not to participate or to withdraw. West Virginia University's Institutional Review Board approval of this project is on file.

I hope that you will participate in this research project, as it could be beneficial in understanding mindfulness, perfectionism, and performance among collegiate gymnasts. Thank you very much for your time. Should you have any questions about this letter or the research project, please feel free to contact Erika Van Dyke by phone at (916) 995-3120, or by email at edv0001@mix.wvu.edu.

Thank you for your time and help with this project.

Sincerely, Erika Van Dyke 
Appendix F

IRB Approval

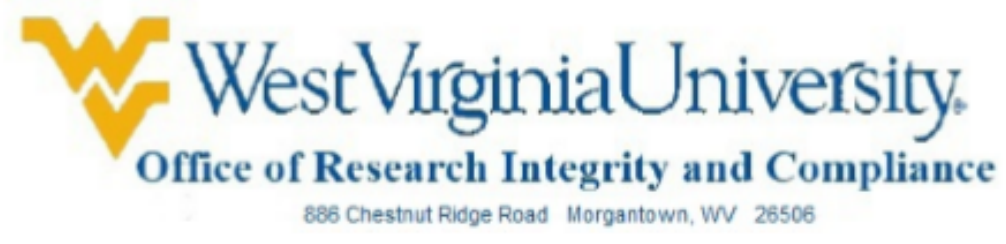

Approval of Human Research Protocol

$08 / 07 / 2018$

To: Samuel Zizzi

From: WVU Office of Research Integrity \& Compliance

Protocol Type: Expedited

Submission Type: Initial

Funding: N/A

WVU Protocol \#: 1805118302
Approval Date: 08/07/2018

Expiration Date: 08/06/2019

Protocol Title: Being Mindful of Perfectionism and Performance in Judged Sport Athletes

The West Virginia University Institutional Review Board has reviewed and granted your request for approval of Expedited protocol 1805118302, in accordance with the Federal regulations 45 CFR 46, 21 CFR 50, and 21 CFR 56 (when applicable). Additional details concerning the review are below:

- Category 7. Research on individual or group characteristics or behavior (including, but not limited to, research on perception, cognition, motivation, identity, language, communication, cultural beliefs or practices, and social behavior) or research employing survey, interview, oral history, focus group, program evaluation, human factors evaluation, or quality assurance methodologies. [NOTE: Some research in this category may be exempt from the DHHS regulations for the protection of human subjects. See Exempt Categories and 45 CFR 46.101(b)(2) and (b)(3). This listing refers only to research that is not exempt.]

The following documents were reviewed and approved for use as part of this submission. Only the documents listed below may be used in the research. Please access and print the files in the Notes \& Attachments section of your approved protocol.

- Cover Letter - Revised.docx

- Coach Email Message.docx

- Athlete Mindfulness Questionnaire English Version.doc 
- Sport-MPS-2 (42 items FINAL).doc

WVU IRB approval of protocol 1805118302 will expire on 08/06/2019.

If any study related activities are to continue beyond the expiration date, a renewal application should be submitted no later than four (4) weeks prior to the expiration date. It is your responsibility to submit your protocol for continuing review.

Once you begin your human subjects research, the following regulations apply:

1. Unanticipated or serious adverse events and/or side effects encountered in this research study must be reported to the IRB within five (5) days using the Notify IRB action in the electronic protocol.

2. Any modifications to the study protocol or informed consent form must be reviewed and approved by the IRB prior to implementation. These modifications should be submitted as an amendment.

3. You may not use a modified informed consent form until it has been reviewed and approved by the WVU IRB. Only consent forms with the WVU+kc watermark may be used to obtain informed consent from participants.

The Office of Research Integrity and Compliance will be glad to provide assistance to you throughout the research process. Please feel free to contact us by phone, at 304.293 .7073 or by email at IRB@mail.wvu.edu.

Sincerely,

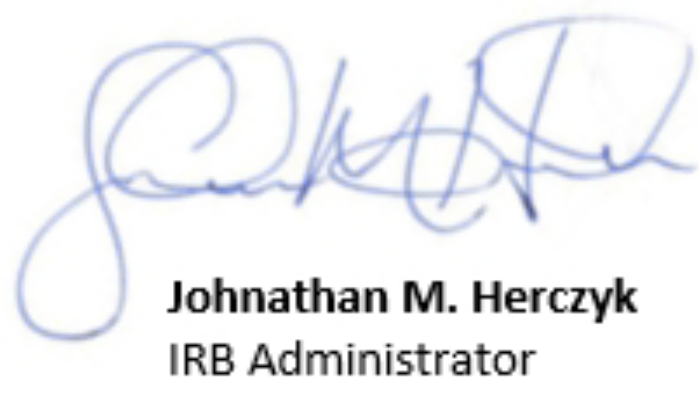

